\title{
RADIATION OF SEISMIC SURFACE-WAVES FROM FINITE MOVING SOURCES
}

\author{
By Ari Ben-Menahem
}

\begin{abstract}
A theory is proposed for the propagation of seismic surface-waves from finite moving sources. The method consists of obtaining, in the first place, basic solutions for surface displacements from directional sources. These solutions are integrated to obtain the effect of a moving fault with arbitrary dip angle. Displacements are evaluated for Rayleigh and Love waves at long ranges. It is shown that the dimensions of the source and the speed of rupture play an important role in the wave-pattern and cannot be ignored whenever the dimensions of the source are of the order of the radiation's dominant wave-length. It is demonstrated how this theory may lead to a derivation of the velocity of rupture and the length of faulting from seismic records of a single station.
\end{abstract}

\section{List of Symbols}

$a_{j} \quad$ defined in equation (1-79)

$b$ the extension of the fault in the direction of motion

$B_{m} \quad$ cylinder function of order $m$

$$
\begin{aligned}
& C_{1}=C_{31} \frac{\sqrt{\gamma^{2}-1}}{\gamma}, \text { where }\left(2 C_{31}\right)^{-1}=\frac{16}{\gamma}\left\{\sqrt{\left(\gamma^{2}-1\right)\left(\gamma^{2}-\frac{1}{3}\right)}-\frac{\left(\gamma^{4}-\gamma^{2}+\frac{1}{3}\right.}{\left(\gamma^{2}-\frac{1}{2}\right)}\right\} \\
& C_{2}=C_{31} \frac{\sqrt{\gamma^{2}-1}}{\gamma^{2}} \\
& C_{3}=C_{31} \frac{\sqrt{\left(\gamma^{2}-1\right)\left(\gamma^{2}-\frac{1}{3}\right)}}{\gamma^{2}-\frac{1}{2}} \\
& C_{4}=C_{3} \frac{\sqrt{\gamma^{2}-\frac{1}{3}}}{\gamma} \\
& C_{1}^{\prime}=C_{1} \sqrt{\frac{2}{\pi \gamma}} \\
& C_{2}^{\prime}=C_{2} \sqrt{\frac{2}{\pi \gamma}} \\
& C_{3}^{\prime}=C_{3} \sqrt{\frac{2}{\pi \gamma}} \\
& C_{4}^{\prime}=C_{4} \sqrt{\frac{2}{\pi \gamma}} \\
& C_{5}^{\prime}=C_{I} \sqrt{\frac{\gamma}{\gamma^{2}-1}} C_{R}^{-1 / 2} \\
& C_{6}^{\prime}=2 C_{1} \frac{\pi}{\sqrt{\gamma}} C_{R}^{-3 / 2}
\end{aligned}
$$

Manuscript received for publication October 7, 1960 and December 14, 1960, and, as revised, May 4, 1961. 


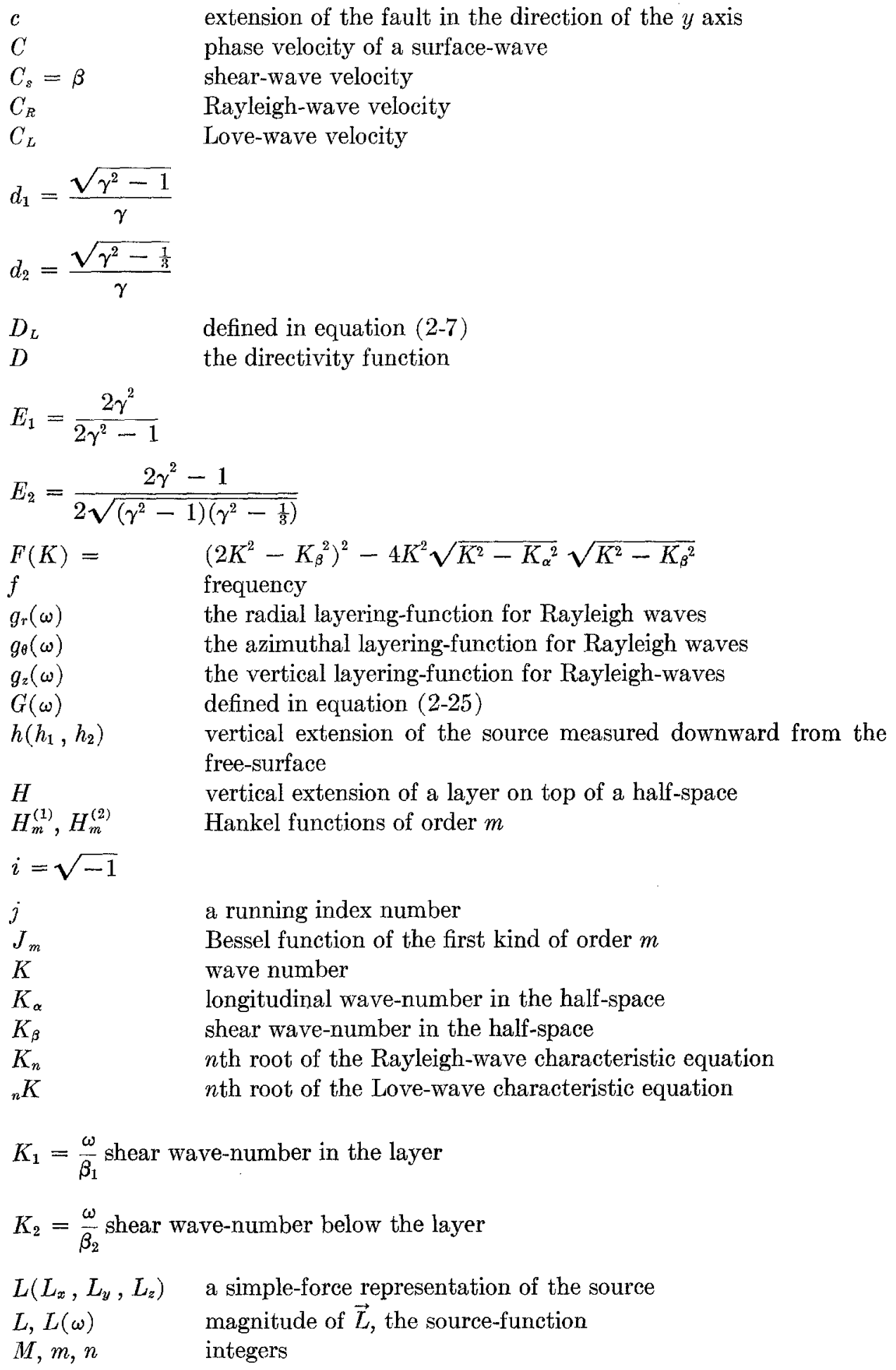




$$
\begin{aligned}
& M_{1}=C_{1} \sqrt{\frac{2 \gamma}{\pi\left(\gamma^{2}-1\right)}} \\
& M_{2}=C_{2} \sqrt{\frac{2 \gamma}{\pi\left(\gamma^{2}-1\right)}} \\
& M_{3}=C_{3} \sqrt{\frac{2 \gamma}{\pi\left(\gamma^{2}-1\right)}} \\
& N_{0}, N_{1} \quad \text { defined in equations (2-12) and (2-23) } \\
& P_{n} \quad \begin{array}{ll}
\text { the polar phase-shift of surface-wave of order } n \\
Q^{-1} & \text { a parameter of the source defined in equation (2-31) } \\
r, r_{0} & \text { the dimensionless dissipation parameter of the Earth } \\
(s, q) & \text { the cartesian components of the wave-number vector } \\
t & \text { time } \\
T & \text { coriod } \\
\left(u_{x}, u_{y}, u_{z}\right) & \text { components of the displacement vector in cartesian coordinates } \\
\left(u_{r}, u_{\theta}, u_{z}\right) & \text { components of the displacement vector in cylindrical coordinates } \\
\left(U_{r}^{R}, U_{\theta}{ }^{R}, U_{z}{ }^{R}\right) & \text { the integrated Raleigh-wave displacement } \\
\left(U_{r}{ }^{L}, U_{\theta}{ }^{L}, 0\right) & \text { the integrated Love-wave displacement } \\
U_{g} & \text { group velocity } \\
U_{0} & \text { a constant value of the group velocity } \\
U_{m} & \text { a stationary value of the group velocity } \\
v & \text { the rupture-velocity } \\
x, y, z & \text { cartesian coordinates }
\end{array} \\
& \begin{array}{ll}
Y_{R}=\frac{\omega}{2 C_{R}} \cos \theta_{0} &
\end{array}
\end{aligned}
$$

a complex number

$$
\begin{aligned}
& \alpha_{1}=\sqrt{\frac{1}{\beta_{1}{ }^{2}}-\frac{1}{C_{L}{ }^{2}}} \\
& \alpha_{2}=\sqrt{\frac{1}{C_{L}{ }^{2}}-\frac{1}{\beta_{2}{ }^{2}}}
\end{aligned}
$$

$\begin{array}{lc}\bar{\alpha} & \text { defined in section } 2.6 \\ \alpha & \text { dilatational velocity in the half-space } \\ \alpha_{0}=6 \times 10^{-4} \mathrm{sec}^{-1} & \text { shear velocity in the half-space } \\ \beta & \text { shear velocity in the layer } \\ \beta_{1} & \text { shear velocity below the layer } \\ \beta_{2} & \text { defined in section } 2.6 \\ \bar{\beta} & \text { the radius of the Earth } \\ \gamma=\frac{1}{2} \sqrt{3+\sqrt{3}} & =1.087664 \\ \Gamma_{0} & \text { the Dirac delta-function } \\ \delta(t) & \text { dip angle } \\ \delta & \text { the initial phase } \\ \delta_{0} & \end{array}$


$\Delta_{n}$

$\Delta h$

$\zeta=\frac{\mu_{2}}{\mu_{1}}$

$\mathrm{Z}_{R}=\frac{\omega c}{2 C_{R}} \sin \theta_{0}$

$\eta$

$\theta, \theta_{0}$

$\Theta$

$\Theta_{1}, \Theta_{2}$

$\bar{\lambda}$

$\lambda$

$\Lambda_{1}^{R}, \Lambda_{2}{ }^{R}$

$\Lambda_{1}{ }^{L}, \Lambda_{2}{ }^{L}$

$\mu$

$\mu_{1}$

$\mu_{2}$

$\nu=\sqrt{\overline{K^{2}}-K_{\alpha}^{2}}$

$\nu_{\perp}=\sqrt{K^{2}-K_{1}^{2}}$

$\xi$

$\sigma$

$\bar{\sigma}$

$\tau$

$\Upsilon_{1}=\frac{\gamma^{2}}{\gamma^{2}-\frac{1}{2}} \sqrt{\frac{\gamma^{2}-1}{\gamma^{2}-\frac{1}{3}}}=0.8038$

$\Upsilon_{2}=\frac{\gamma^{2}-\frac{1}{2}}{\gamma^{2}-\frac{1}{3}}=0.8003$

$$
\begin{aligned}
& \varphi_{n} \quad \text { defined in equation (3-7) } \\
& \varphi_{R} \quad \text { defined in equation (1-41) } \\
& \varphi_{L} \quad \text { defined in equation (2-19) } \\
& \partial_{1} \varphi \quad \text { differential phase of the first order } \\
& \partial_{2} \varphi \text { differential phase of the second order } \\
& \phi_{1,2} \quad \text { longitudinal potential function } \\
& x=\operatorname{tg}^{-1} \frac{q}{s} \\
& \mathrm{X}_{R}=\frac{\omega b}{2 C_{R}}\left(\frac{C_{R}}{v}-\cos \theta_{0}\right) \quad \mathrm{X}_{L}=\frac{\omega b}{2 C_{L}}\left(\frac{C_{L}}{v}-\cos \theta_{0}\right) \\
& \underset{\rightarrow}{\psi_{R}} \quad \text { defined in equation (1-52) } \\
& \vec{\Psi} \quad \text { vector potential function } \\
& \Omega \quad \text { defined in section } 2.6 \\
& \omega\left(\omega_{1}, \omega_{2}\right) \quad \text { angular frequency } \\
& \bar{\omega} \quad \text { average value of } \omega \text { over a spectral window }
\end{aligned}
$$

integration variable

polar angle on the free-surface

Lame parameter

defined in equation (1-76)

defined in equation (2-20)

igidity in the half-space

rigidity in the layer

$\nu^{\prime}=\sqrt{K^{2}-K_{\beta}^{2}}$

integration variable

extension of strike-slip fault in the dip direction

extention of dip-slip fault in the dip direction defined in equation (1-55) 


\section{Chapter 1-Rayleigh Waves from Finite Moving Sources}

\subsection{INTRODUCTION}

There is no doubt that seismic records carry information about the earthquake source, such as its dimensions, the speed of rupture and other parameters of interest. The point-source model is not sufficient for these purposes and one has to consider the finiteness of the source if one wishes to be able to interpret details of amplitude variation on a seismogram. Early attempts in this direction were made by Lamb (1916) and Sezawa (1929). Sezawa studied an extended source in the form of an infinite sheet. His results are of little value to our case, since he derived his results for infinite plane of sources and no propagating disturbance.

Knopoff and Gilbert (1959) used the powerful "Knopoff-deHoop representation theorem" to obtain first motions of body-waves from a disturbance propagating along a line. However, using their method for finite regions incorporates unsurmountable integrations, even for the simplest cases, which obviously renders the method ineffective, except for initial motions.

In this chapter we investigate the effect of the finiteness of the seismic focus on the Rayleigh-wave pattern. The equations of motion are solved for an internal harmonic concentrated force which points in an arbitrary direction. A fault plane is then realized by moving this source along a line with finite speed and integrating the Rayleigh-pole contribution across a finite rectangle with an arbitrary strike and dip. Displacements are evaluated for long ranges and expressions are obtained for strike-slip and dip-slip fault types. Attention is mainly focused on a couple type motion of a vertical strike-slip model for which displacements have been actually computed and the results transformed into the time domain. It is found that the finiteness of the source plays a dominant role in the wave pattern whenever the wave-length is of the order of the fault dimensions or when the time of rupture is of the order of the period. It is also found that azimuthal distribution of amplitudes in that range depends strongly on the dimensions of the source and that the energy radiated in the direction of motion may highly exceed the amount radiated in the opposite direction. Not all possible faulting models are studied, but the method used is readily applicable to any special case. The radiation of $P$ and $S$ waves from moving faults will be treated in another paper.

\subsection{INTEGRAL REPRESENTATION OF THE DISPLACEMENTS DUE TO A HORIZONTAL FORCE}

Consider an elastic half-space with a coordinate system as shown in figure 1 . At the point $x=0, y=0, z=h$, we put a harmonic point-source of magnitude $\mathrm{L}$ which is a simple force pointing in the direction of the $x$-axis. Following a method given by Yanovskaya (1958) we describe this source in the form of a double Fourierintegral (Morse and Feshbach 1953)

$$
\begin{aligned}
& P_{x z}=\frac{L}{2 \pi} e^{i \omega t} \int_{-\infty}^{\infty} \int_{-\infty}^{\infty} e^{i(s x+q y)} d s d q \\
& P_{y z}=0 \\
& P_{z z}=0
\end{aligned}
$$


To evaluate the displacement field over the free surface of the half space we have to solve the wave equations:

$$
\begin{array}{rlr}
\nabla^{2} \phi_{i} & =\frac{1}{\alpha^{2}} \frac{\partial^{2} \phi_{i}}{\partial t^{2}} & \\
\nabla^{2} \vec{\Psi}_{i} & =\frac{1}{\beta^{2}} \frac{\partial^{2} \vec{\Psi}_{i}}{\partial t^{2}} & i=1,2
\end{array}
$$

where $\phi$ is the dilatation potential, $\vec{\Psi}\left(\Psi^{(x)}, \Psi^{(y)}, \Psi^{(z)}\right)$ is the shear potential and the

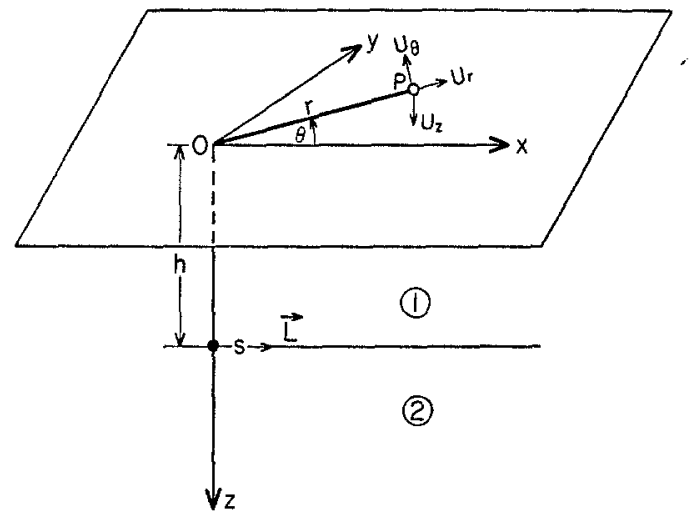

FIG. 1. Initial stress in the $x$-direction, source buried at depth $h$ below the free surface. $r$ and $\theta$ are polar coordinates of the point of observation.

subscripts 1, 2 refer to the region above and below the source, respectively. We shall seek solutions of the form:

$$
\left.\begin{array}{rl}
\phi_{1} & =\frac{L}{2 \pi} e^{i \omega t} \int_{-\infty}^{\infty} \int_{-\infty}^{\infty}\left(A_{1} \operatorname{ch\nu } z+B_{1} s h \nu z\right) e^{i(s x+q y)} d s d q \\
\Psi_{1}^{(x)} & =\frac{L}{2 \pi} e^{i \omega t} \int_{-\infty}^{\infty} \int_{-\infty}^{\infty}\left(C_{1}^{(x)} c h \nu^{\prime} z+D_{1}^{(x)} s h \nu^{\prime} z\right) e^{i(s x+q y)} d s d q \\
\Psi_{1}^{(y)} & =\frac{L}{2 \pi} e^{i \omega t} \int_{-\infty}^{\infty} \int_{-\infty}^{\infty}\left(C_{1}^{(y)} c h \nu^{\prime} z+D_{1}^{(y)} s h \nu^{\prime} z\right) e^{i(s x+q y)} d s d q
\end{array}\right)<h
$$

The displacements above the source are expressed in terms of these potentials 
(Ewing, Jardetzky, and Press 1957):

$$
\begin{aligned}
& u_{x}=\frac{\partial \phi_{1}}{\partial x}-\frac{\partial \Psi_{1}^{(y)}}{\partial z} \\
& u_{y}=\frac{\partial \phi_{1}}{\partial y}+\frac{\partial \Psi_{1}^{(x)}}{\partial z} \\
& u_{z}=\frac{\partial \phi_{1}}{\partial z}+\frac{\partial \Psi_{1}^{(y)}}{\partial x}-\frac{\partial \Psi_{1}^{(x)}}{\partial y}
\end{aligned}
$$

Likewise, the stresses are expressed in terms of the potentials via the following relations (Ewing, Jardetzky, and Press 1957):

$$
\begin{aligned}
& P_{x z}=\mu\left(\frac{\partial u_{x}}{\partial z}+\frac{\partial u_{z}}{\partial x}\right) \\
& P_{y z}=\mu\left(\frac{\partial u_{z}}{\partial y}+\frac{\partial u_{y}}{\partial z}\right) \\
& P_{z z}=2 \mu \frac{\partial u_{z}}{\partial z}+\bar{\lambda} \operatorname{div} \vec{u}
\end{aligned}
$$

We express both the stresses and the displacements in terms of the potentials and subject them to the boundary conditions:

$$
\left.\begin{array}{rrrl}
P_{x z}=0 ; & P_{y z}=0 ; & P_{z z}=0 & \text { at } z=0 \\
u_{x}^{(1)}=u_{x}^{(2)} & P_{x z}^{(1)}-P_{x z}^{(2)}=P_{x z} \\
u_{y}^{(1)}=u_{y}^{(1)} & P_{y z}^{(1)}=P_{y z}^{(2)} \\
u_{z}^{(1)}=u_{z}^{(2)} & P_{z z}^{(1)}=P_{z z}^{(2)}
\end{array}\right\} \quad \text { at } z=h
$$

Thus we obtain nine equations in the nine coefficients $A_{1}, A_{2}, C_{1}^{(x)}, C_{1}^{(y)}, C_{2}^{(x)}$, $C_{2}^{(y)}, D_{1}^{(x)}, D_{1}^{(y)}, B_{1}$. After solving these in terms of $s, q, \nu$ and $\nu^{\prime}$ we substitute the relevant coefficients into the expressions for the surface displacements which were obtained by substituting the set (1-3) into (1-4), (1-5) and (1-6):

$$
\begin{aligned}
& u_{x}=\frac{L}{2 \pi} e^{i \omega t} \int_{-\infty}^{\infty} \int_{-\infty}^{\infty}\left(i s A_{1}-\nu^{\prime} D_{1}^{(y)}\right) e^{i(s x-q y)} d s d q \\
& u_{y}=\frac{L}{2 \pi} e^{i \omega t} \int_{-\infty}^{\infty} \int_{-\infty}^{\infty}\left(i q A_{1}+\nu^{\prime} D_{1}^{(x)}\right) e^{i(s x+q y)} d s d q \\
& u_{z}=\frac{L}{2 \pi} e^{i \omega t} \int_{-\infty}^{\infty} \int_{-\infty}^{\infty}\left({ }_{\nu} B_{1}+i s C_{1}^{(y)}-i q C_{1}^{(x)}\right) e^{i(s x+q y)} d s d q
\end{aligned}
$$

We now change from the Fourier transform to the Hankel transform by the substitutions (Sneddon 1950):

$$
s+i q=K e^{i x} \quad x+i y=r e^{i \theta}
$$

and then make use of some integral representations of the Bessel functions (Morse and Feshbach 1953). 


$$
\begin{aligned}
& J_{0}(K r)=\frac{1}{2 \pi} \int_{0}^{2 \pi} e^{i K r \cos (\chi-\theta)} d \chi \\
& J_{1}(K r)=\frac{1}{2 \pi} \int_{0}^{2 \pi} e^{i K r \cos (\chi-\theta)+i\left(\chi-\theta-\frac{\pi}{2}\right)} d \chi
\end{aligned}
$$

to arrive at the expressions for the components of the displacement vector in cylindrical coordinates, $r, \theta, z$ at $z=0$ :

$$
\begin{aligned}
& u_{r}=\frac{L \cos \theta}{2 \pi \mu} \\
& \cdot e^{i \omega t} \int_{0}^{\infty} \frac{K \nu^{\prime}\left\{\left(2 K^{2}-K_{\beta}{ }^{2}\right) e^{-\nu^{\prime} h}-2 K^{2} e^{-\nu h}\right\}}{F(K)}\left[J_{0}(K r)-\frac{1}{K r} J_{1}(K r)\right] d K \\
& u_{\theta}=\frac{L \sin \theta}{2 \pi \mu r} e^{i \omega t} \int_{0}^{\infty} \frac{\left(2 K^{2}-K_{\beta}{ }^{2}\right) e^{-\nu^{\prime} h}-2 K^{2} e^{-\nu h}}{F(K)} \nu^{\prime} J_{1}(K r) d K \\
& u_{z}=\frac{L \cos \theta}{2 \pi \mu} e^{i \omega t} \int_{0}^{\infty} \frac{\left(2 K^{2}-K_{\beta}{ }^{2}\right) e^{-\nu h}-2 \nu \nu^{\prime} e^{-\nu^{\prime} h}}{F(K)} K^{2} J_{1}(K r) d K
\end{aligned}
$$

\subsection{EVALUATTON OF THE RAYLEIGH DISPLACEMENTS}

In (1-18) to $(1-20)$, we replace $2 J_{m}(K r)$ by $H_{m}{ }^{(1)}(K r)+H_{m}{ }^{(2)}(K r)$ and integrate in the complex $K$ plane as shown in figure 2. It is well known (Pekeris 1955) that the residue at the Rayleigh pole at $+\gamma K_{\beta}\left(\gamma=\frac{1}{2} \sqrt{3+\sqrt{3}}\right)$ yields the surface-wave displacements and is given, e.g. for (1-20) in the form

$$
\begin{aligned}
& u_{z}= \frac{L \cos \theta}{2 \pi \mu} e^{i \omega t} \\
& \cdot \frac{\left(2 K_{n}{ }^{2}-K_{\beta}{ }^{2}\right) e^{-h \sqrt{K_{n}^{2}-K_{\alpha^{2}}}}-2 \sqrt{\left(K_{n}{ }^{2}-K_{\alpha}{ }^{2}\right)\left(K_{n}{ }^{2}-K_{\beta}^{2}\right)} e^{-h \sqrt{K_{n}^{2}-K_{\beta^{2}}}}}{\frac{\partial F\left(K_{n}\right)}{\partial K_{n}}} \\
& \cdot K_{n}{ }^{2} H_{1}{ }^{(2)}\left(K_{n} r\right) \cdot \pi i
\end{aligned}
$$

where $K_{n}=\gamma K_{\beta}$ and $\pi i$ arises from taking the principal value at the pole. The final results for all the components of the displacement are:

$$
\begin{aligned}
& u_{r}=i \frac{L}{\mu} K_{\beta} C_{1} \cos \theta e^{i \omega t}\left\{e^{-h K_{n} d_{1}}-E_{1} e^{-h K_{n} d_{2}}\right\} \\
& \cdot\left\{H_{0}{ }^{(2)}\left(K_{n} r\right)-\frac{1}{K_{n} r} H_{0}{ }^{(2)}\left(K_{n} r\right)\right\} \\
& u_{\theta}=i \frac{L}{\mu} C_{2} \sin \theta e^{i \omega t} \cdot \frac{1}{r}\left\{e^{-h K_{n} d_{1}}-E_{1} e^{-h K_{n} d_{2}}\right\} H_{1}{ }^{(2)}\left(K_{n} r\right) \\
& u_{z}=i \frac{L}{\mu} K_{\beta} C_{3} \cos \theta e^{i \omega t}\left\{-e^{-h K_{n} d_{1}}+E_{2} e^{-h K_{n} d_{2}}\right\} H_{1}^{(2)}\left(K_{n} r\right)
\end{aligned}
$$

where $C_{1}, C_{2}, C_{3}$ are positive real constants, $d_{1}=\left(\sqrt{\gamma^{2}-1}\right) / \gamma d_{2}=\left(\sqrt{\gamma^{2}-\frac{1}{3}}\right) / \gamma$, $C_{s}$ is the shear velocity and $C_{R}$ the Rayleigh-wave velocity. Neglecting terms in 
$1 / r$ and using the known approximation (Ryshik and Gradstein 1957), when $|z| \gg|m|$

$$
H_{m}{ }^{(2)}(g)=\sqrt{\frac{2}{\pi_{z}}} e^{-i(z-(m \pi / 2)-(\pi / 4))}\left\{1+0\left(|z|^{-1}\right)\right\}
$$

we get

$$
\begin{aligned}
& u_{r}=\frac{L}{\mu} C_{1}^{\prime} \frac{\cos \theta}{\sqrt{r}} \sqrt{K_{\beta}}\left\{e^{-h K_{n} d_{1}}-E_{1} e^{-h K_{n} d_{2}}\right\} e^{i\left(\omega t-K_{n} r+(3 \pi / 4)\right)} \\
& u_{\theta}=\frac{L}{\mu} C_{2}{ }^{\prime} \frac{\sin \theta}{r \sqrt{r}} \frac{1}{\sqrt{K_{\beta}}}\left\{e^{-h K_{n} d_{1}}-E_{1} e^{-h K_{n} d_{2}}\right\} e^{i\left(\omega t-K_{n} r-(3 \pi / 4)\right)}
\end{aligned}
$$

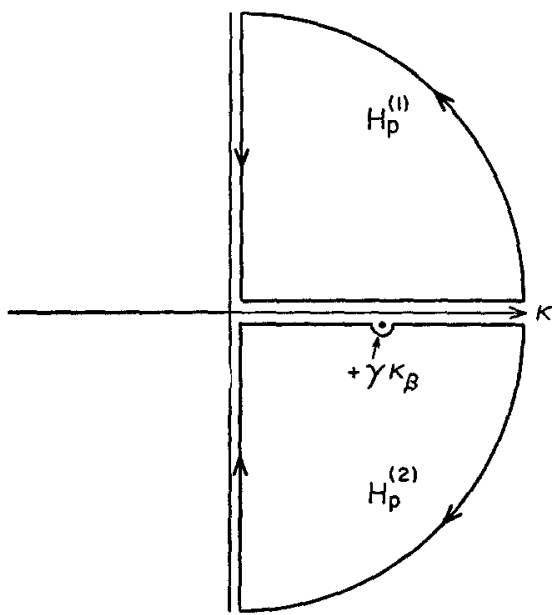

FIG. 2. Path of integration in the complex $k$ plane.

$$
u_{2}=\frac{L}{\mu} C_{3}{ }^{\prime} \frac{\cos \theta}{\sqrt{r}} \sqrt{K_{\beta}}\left\{e^{-h K_{n} d_{1}}-E_{2} e^{-h K_{n} d_{2}}\right\} e^{i\left(\omega t-K_{n} r+\pi / 4\right)}
$$

with $E_{1}=\frac{2 \gamma^{2}}{2 \gamma^{2}-1} ; E_{2}=\frac{2 \gamma^{2}-1}{2 \sqrt{\left(\gamma^{2}-1\right)\left(\gamma^{2}-\frac{1}{3}\right)}}$ and $C_{1}^{\prime}, C_{2}^{\prime}, C_{3}^{\prime}$ positive real constants.

\subsection{A MODEL FOR A VERTICAL STRIKE-SLIP FAULT}

Consider now our previous point source, situated at depth $h$ below the free surface, whose Rayleigh displacements are given by (1-22) to (1-24). Suppose that we have similiar sources distributed continuously from $z=h_{1}$ to $z=h_{2}$ (see fig. 3 ) thus filling the interval $\Delta h$ on the $z$ axis. Let these sources act simultaneously: the integrated effect at a point $P$ (situated on the free surface) for $u_{z}$, say, is, from (1-24):

$$
u_{z}=\frac{L}{\mu} i C_{3} K_{\beta} \cos \theta e^{i \omega t} H_{1}^{(2)}\left(K_{n} r\right) \frac{1}{\Delta h} \int_{h_{1}}^{h_{2}}\left(e^{-h K_{n} d_{1}}-E_{2} e^{-h K_{n} d_{2}}\right) d h
$$


(since there is no phase effect due to the differences in depth)

$$
\begin{array}{r}
u_{z}=\frac{i L C_{3}}{\gamma d_{1} \mu} \cos \theta e^{i \omega t} H_{1}{ }^{(2)}\left(K_{n} r\right) \\
\cdot \frac{\left(e^{-h_{1} K_{n} d_{1}}-e^{-h_{2} K_{n} d_{1}}\right)-\Upsilon_{2}\left(e^{-h_{1} K_{n} d_{2}}-e^{-h_{2} K_{n} d_{2}}\right)}{\Delta h}
\end{array}
$$

The reason for introducing the factor $1 / \Delta h$ in (1-29) is to enable us to fall back on the expression for the point-source as $h_{2} \rightarrow h_{1}$.

Assume further that this segment of sources $\Delta h$, starts at some time $t_{0}=0$ to move to the right (fig. 3 ), in the plane $x z$, with a finite speed $v$, up to the coordi-

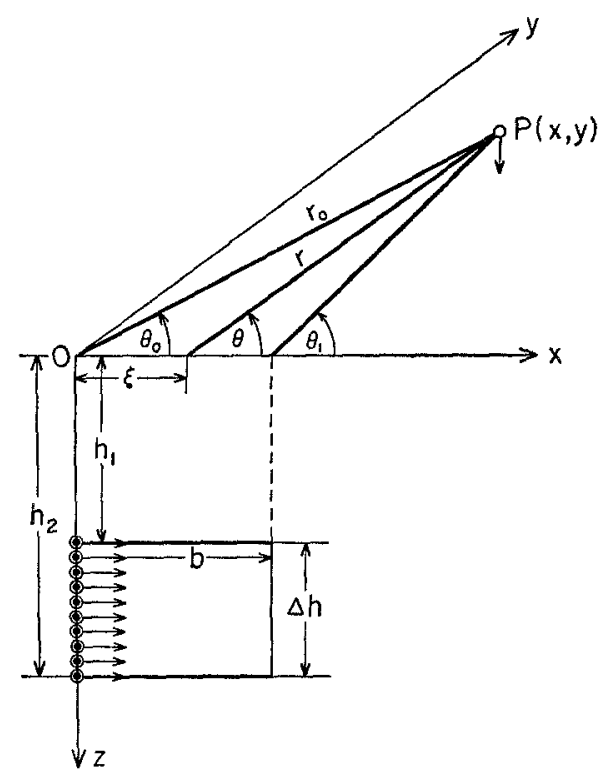

Fig. 3. Realization of fault-plane.

nate $x=b$, radiating as it moves. Taking into consideration the variation both in $r$ and $t$, we get for the $u_{z}$ component:

$$
U_{z}{ }^{R}=i \frac{C_{3}}{d_{1}} \frac{2}{M_{3}} g_{z}(\omega) \frac{1}{b} \int_{0}^{b} \cos \theta H_{1}^{(2)}\left(K_{n} r\right) e^{i \omega(t-\xi / v)} d \xi
$$

with

$$
\begin{gathered}
r^{2}=y^{2}+(x-\xi)^{2} ; \quad \cos \theta=(x-\xi) r^{-1} \\
g_{z}=\frac{M_{3} L}{2 \mu \Delta h}\left\{\left(e^{-h_{1} K_{n} d_{1}}-e^{-h_{2} K_{n} d_{1}}\right)-\Upsilon_{2}\left(e^{-h_{1} K_{n} d_{2}}-e^{-h_{2} K_{n} d_{2}}\right)\right\}
\end{gathered}
$$

In order to evaluate (1-31) we make use of the multiplication formulae of the Bessel functions (Ryshik and Gradstein 1957):

$$
B_{m}(\vartheta R)=\vartheta^{m} \sum_{M=0}^{\infty} \frac{B_{m+M}(R)}{M !}\left[\frac{\left(1-\vartheta^{2}\right)}{2} R\right]^{M} \quad\left|1-\vartheta^{2}\right|<1
$$


The geometry in figs. 3 and 4 yields

$$
R=r_{0} K_{n} \text { and } r=r_{0} \sqrt{1-2\left(\xi / r_{0}\right) \cos \theta_{0}+\left(\xi^{2} / r_{0}^{2}\right)} .
$$

We choose $\vartheta^{2}=1-2\left(\xi / r_{0}\right) \cos \theta_{0}+\left(\xi^{2} / r_{0}^{2}\right)$ so that

$$
\left|1-\vartheta^{2}\right|=\left|\xi / r_{0}\left(2 \cos \theta_{0}-\left(\xi / r_{0}\right)\right)\right|<1 \text { for }\left(\xi / r_{0}\right)<1 .
$$

Substituting this into (1-32), and (1-32) into (1-31), and neglecting terms of the order $\left(\xi / r_{0}\right)^{2}$, we obtain for the integral (which we denote $J$ for brevity),

$$
b J=e^{i \omega t} \int_{0}^{b} \vartheta \cos \theta\left\{\sum_{M=0}^{\infty} \frac{1}{M !}\left(K_{n} \xi \cos \theta\right)^{M} H_{1+M}^{(2)}\left(K_{n} r_{0}\right)\right\} e^{-i \omega(\xi / v)} d \xi
$$

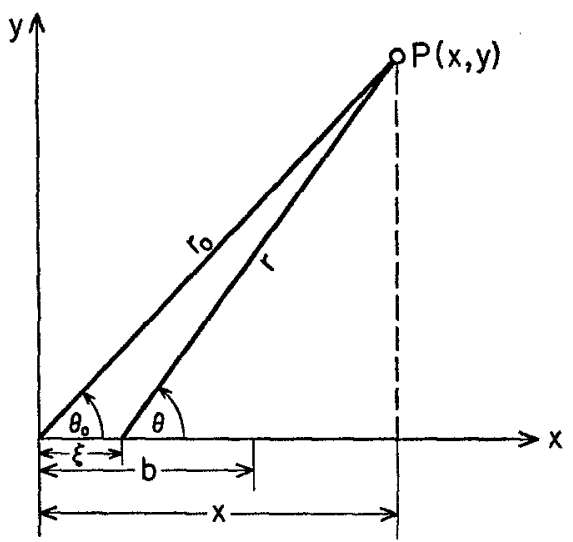

Hence,

FIG. 4. Geometry of free surface.

$J=\frac{\cos \theta_{0}}{\sqrt{r_{0}}} \sqrt{\frac{2}{\pi K_{n}}} \frac{e^{i K_{n} b\left(\cos \theta_{0}-C_{R} / v\right)}-1}{K_{n} b\left(\cos \theta_{0}-\left(C_{R} / v\right)\right)} e^{i\left(\omega t-K_{n}{ }^{r}+(3 \pi / 4)\right)}+0\left[\left(K_{n} r_{0}\right)^{-3 / 2}\right]$

where terms higher than $1 / \sqrt{r_{0}}$ have been neglected in accordance with (1-25). In addition it is assumed that the extension of the source is small as compared with the range $r_{0}$. These assumptions are summed up as:

$$
\begin{aligned}
\frac{\omega r_{0}}{C_{R}} \gg 1 \\
\left(\frac{b}{r_{0}}\right) \ll 1
\end{aligned}
$$

Simplifying equation (1-34) and repeating the former technique for the other components, we obtain the parallel expressions for $U_{r}{ }^{R}$ and $U_{\theta}{ }^{R}$; the results are:

$$
\begin{aligned}
U_{r}^{R} & =\frac{2 \cos \theta_{0}}{\sqrt{K_{\beta} r_{0}}} g_{r}(\omega)\left\{\frac{\sin \mathrm{X}_{R}}{\mathrm{X}_{R}}\right\} e^{i\left(\varphi_{R}-(\pi / 4)\right)} \\
U_{\theta}{ }^{R} & =\frac{\sin \theta_{0}}{\left(K_{\beta} r_{0}\right)^{3 / 2}} g_{\theta}(\omega)\left\{\frac{\sin \mathrm{X}_{R}}{\mathrm{X}_{R}}\right\} e^{i\left(\varphi_{R}+(\pi / 4)\right)} \\
U_{z}{ }^{R} & =\frac{2 \cos \theta_{\theta}}{\sqrt{K_{\beta} r_{0}}} g_{z}(\omega)\left\{\frac{\sin \mathrm{X}_{R}}{\mathrm{X}_{R}}\right\} e^{i\left(\varphi_{R}-(3 \pi / 4)\right)}
\end{aligned}
$$


where $M_{i}$ are some real positive constants and

$$
\begin{gathered}
\mathrm{X}_{R}=\frac{\omega b}{2 C_{R}}\left(\frac{C_{R}}{v}-\cos \theta_{0}\right) \\
\varphi_{R}=\omega\left(t-\frac{r_{0}}{C_{R}}\right)-\mathrm{X}_{R} \\
g_{r}(\omega)=\frac{M_{1}}{2 M_{2}} g_{\theta}(\omega) \\
=\frac{M_{1} L}{2 \mu \Delta h \gamma}\left\{\left(e^{-h_{1} K_{n} d_{1}}-e^{-h_{2} K_{n} d_{1}}\right)-\Upsilon_{1}\left(e^{-h_{1} K_{n} d_{2}}-e^{-h_{2} K_{n} d_{2}}\right)\right\}
\end{gathered}
$$

The ratio $C_{R} / v$ is not subjected to any theoretical limitations.

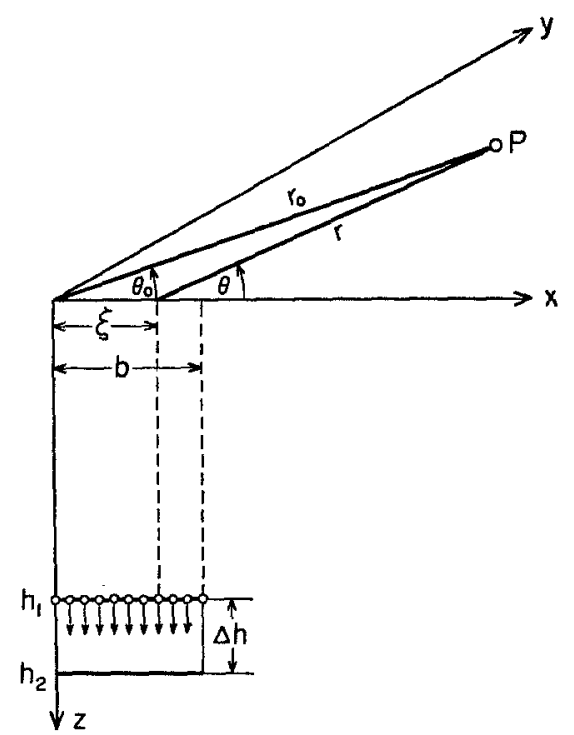

FIG. 5. Geometry of vertical dip-slip fault.

\subsection{A MODEL FOR A VERTICAL DIP-SLIP FAULT}

We shall now calculate the Rayleigh displacements for the case of a vertical dip-slip fault (fig. 5). We shall make use of the results given by Pekeris (1955) for a vertical concentrated point-source. Transforming his results (which were originally given for a step time-dependence of the source) back into the steady-state source, we find:

$$
\begin{aligned}
& u_{r}=\frac{-L}{2 \pi \mu} e^{i \omega t} \int_{0}^{\infty} \frac{2 \nu \nu^{\prime} e^{-\nu h}-\left(2 K^{2}-K_{\beta}{ }^{2}\right) e^{-\nu^{\prime} h}}{F(K)} J_{1}(K r) K^{2} d K \\
& u_{\theta}=0 \\
& u_{z}=\frac{L}{2 \pi \mu} e^{i \omega t} \int_{0}^{\infty} \frac{\left(2 K^{2}-K_{\beta}^{2}\right) e^{-\nu h}-2 K^{2} e^{-\nu^{\prime} h}}{F(K)} J_{0}(K r) \nu K d K
\end{aligned}
$$


On using the method of section 1.3 we get for the Rayleigh displacements:

$$
\begin{aligned}
& u_{r}=\frac{-i L C_{3}}{\mu} K_{\beta} e^{i \omega t} H_{1}^{(2)}\left(K_{n} r\right)\left\{e^{-h K_{n} d_{2}}-E_{2} e^{-h K_{n} d_{1}}\right\} \\
& u_{z}=\frac{i L C_{4}}{\mu} K_{\beta} e^{i \omega t} H_{0}{ }^{(2)}\left(K_{n} r\right)\left\{e^{-h K_{n} d_{2}}-E_{1} e^{-h K_{n} d_{1}}\right.
\end{aligned}
$$

The realization of the fault plane in this case is done in the following way: a horizontal line of sources which extends from $x=0$ to $x=b$ starts to radiate at some time $t_{0}=0$. This line segment then moves downward in the $z$ direction from $z=h_{1}$ to $z=h_{2}$, with a velocity $v$, radiating as it moves. The computation of the displacement at some point $P(\theta, r)$ on the free surface involves two integrations:

$$
\begin{gathered}
U_{r}{ }^{R}=\frac{i L C_{3}}{\mu b \cdot \Delta h} K_{\beta} \\
\cdot \int_{h_{1}}^{h_{2}} \int_{0}^{b}\left(e^{-h K_{n} d_{2}}-E_{2} e^{-h K_{n} d_{1}}\right) H_{1}^{(2)}\left(K_{n} r\right) e^{i \omega\left(t-\left(h-h_{1} / v\right)\right)} d h d \xi \\
U_{z}{ }^{R}(\omega)=\frac{i L C_{4}}{\mu b \cdot \Delta h} K_{\beta} \\
\cdot \int_{h_{2}}^{h_{1}} \int_{0}^{b}\left(e^{-h K_{n} d_{1}}-E_{1} e^{-h K_{n} d_{2}}\right) H_{0}^{(2)}\left(K_{n} r\right) e^{-i \omega\left(t-\left(h-h_{1} / v\right)\right)} d h d \xi
\end{gathered}
$$

with

$$
r=r_{0} \sqrt{1-2 \frac{\xi}{r_{0}} \cos \theta_{0}+\frac{\xi^{2}}{r_{0}^{2}}}
$$

Following our previous method, (1-32) to (1-35), we get, to a first approximation:

$$
\begin{aligned}
i e^{i \omega t} \frac{1}{\bar{b}} \int_{0}^{b} H_{0}^{(2)}\left(K_{n} r\right) d \xi & =\sqrt{\frac{2}{\pi K_{n} r_{0}}}\left\{\frac{\sin Y_{R}}{Y_{R}}\right\} e^{i\left(\psi_{R}+(3 \pi / 4)\right)} \\
-i e^{i \omega t} \frac{1}{b} \int_{0}^{b} H_{1}^{(2)}\left(K_{n} r\right) d \xi & =\sqrt{\frac{2}{\pi K_{n} r_{0}}}\left\{\frac{\sin Y_{R}}{Y_{R}}\right\} e^{i\left(\psi_{R}+(\pi / 4)\right)}
\end{aligned}
$$

with

$$
Y_{R}=\frac{\omega b \cos \theta_{0}}{2 C_{R}} ; \quad \psi_{R}=\omega\left(t-\frac{r_{0}}{C_{R}}\right)+Y_{R}
$$

The second integration over $h$ is exact. The final results are

$$
\begin{gathered}
U_{z}{ }^{R}=\frac{L C_{4}^{\prime}}{\mu \sqrt{r_{0}}} \sqrt{K_{\beta}} \frac{\sin Y_{R}}{Y_{R}} \\
\cdot\left\{\frac{\sin \Theta_{2}}{\Theta_{2}} e^{i \Theta_{2}-h_{1} K_{n} d_{2}}-E_{1} \frac{\sin \Theta_{1}}{\Theta_{1}} e^{i \Theta_{1}-h_{1} K_{n} d_{1}}\right\} e^{i\left(\psi_{R}+(3 \pi / 4)\right)} \\
U_{r}{ }^{R}=\frac{L C_{3}^{\prime}}{\mu \sqrt{r_{0}}} \sqrt{K_{\beta}} \frac{\sin Y_{R}}{Y_{R}} \\
\cdot\left\{\frac{\sin \Theta_{2}}{\Theta_{2}} e^{i \Theta_{2}-h_{1} K_{n} d_{2}}-E_{2} \frac{\sin \Theta_{1}}{\Theta_{1}} e^{i \Theta_{1}-h_{1} K_{n} d_{1}}\right\} e^{i\left(\psi_{R}+(\pi / 4)\right)}
\end{gathered}
$$


with

$$
\begin{aligned}
& \Theta_{1}=\frac{\left(h_{2}-h_{1}\right) \omega}{2 C_{R}}\left(i \frac{\sqrt{\gamma^{2}-1}}{\gamma}-\frac{C_{R}}{v}\right) \\
& \Theta_{2}=\frac{\left(h_{2}-h_{1}\right) \omega}{2 C_{R}}\left(i \frac{\sqrt{\gamma^{2}-\frac{1}{3}}}{\gamma}-\frac{C_{R}}{v}\right)
\end{aligned}
$$

Note that $\Theta_{1}$ and $\Theta_{2}$ are complex. For the sake of numerical computations it is useful to use the relations:

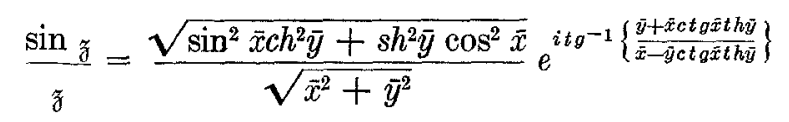

where $z=\vec{x}+i \bar{y}$. When dealing with high frequencies, the previous equations may be simplified due to the fact that $d_{2} \cong 2 d_{1}$ and the term involving $\Theta_{2}$ in (1-53) and (1-54) becomes insignificant. We also observe that in case $C_{R} / v \gg \sqrt{\gamma^{2}-1} / \gamma$, i.e., $v \ll C_{R}, \Theta_{1,2}$ may be taken as real. In case $v \gg C_{R}$, it may be considered pure imaginary. For either long periods, or $b \rightarrow 0$ and $h_{1} \rightarrow h_{2}$ we fall back on the pointsource solution.

\subsection{DIP-SLIP FAULT WITH ARBITRARY DIP ANGLE}

Consider a fault plane striking in the direction of the $y$ axis, and dipping at an angle $\delta$ with respect to the free surface (fig. 6 ). Let a simple force $\vec{L}$, pointing along the fault, be localized at each point of a segment $y=0$ to $y=c$ (which is parallel to the $y$ axis at depth $z=h_{1}$ ). We may split the force $\vec{L}$ into a vertical component and a horizontal component,

$$
\begin{aligned}
& L_{x}=|L| \cos \delta \\
& L_{z}=|L| \sin \delta
\end{aligned}
$$

We shall first compute the three components $U_{r}{ }^{R}, U_{\theta}{ }^{R}$ and $U_{z}{ }^{R}$ due to $L_{x}$. Making use of (1-22) to (1-24), we have:

$$
\begin{aligned}
& \left(U_{r}^{R}\right)_{x}=\frac{i L C_{1}}{\mu \bar{\sigma} C} K_{\beta} \\
& \cdot \int_{0}^{\bar{\sigma}} \int_{0}^{c}\left\{e^{-K_{n} d_{2}\left(h_{1}+\xi \sin \delta\right)}-E_{1} e^{-K_{n} d_{1}\left(h_{1}+\xi \sin \delta\right)}\right\} \cos \theta H_{0}^{(2)}\left(K_{n} r\right) e^{i \omega(t-(\xi / v))} d \eta d \xi \\
& \left(U_{\theta}^{R}\right)_{x}=\frac{i L C_{2}}{\mu \bar{\sigma} c} \\
& \cdot \int_{0}^{\bar{\sigma}} \int_{0}^{c}\left\{e^{-K_{n} d_{2}\left(h_{1}+\xi_{\xi} \operatorname{in} \tilde{o}\right)}-E_{1} e^{-K_{n} d_{1}\left(h_{1}+\xi_{\sin \delta}\right)}\right\} \frac{\sin \theta}{r} H_{1}^{(2)}\left(K_{n} r\right) e^{i \omega(t-(\xi / v))} d \eta d \xi \\
& \left(U_{z}^{R}\right)_{x}=\frac{-i L C_{3}}{\mu \bar{\sigma} c} K_{\beta} \\
& \cdot \int_{0}^{\bar{\sigma}} \int_{0}^{c}\left\{e^{-K_{n} d_{2}\left(h_{1}+\xi_{\sin } \delta\right)}-E_{2} e^{-K_{n} d_{1}\left(h_{1}+\xi \sin \delta\right)}\right\} \cos \theta H_{1}^{(2)}\left(K_{n} r\right) e^{i \omega(i-(\xi / v))} d \eta d \xi
\end{aligned}
$$

with

$$
r^{2}=(x-\xi \cos \delta)^{2}+(y-\eta)^{2}
$$


where $c$ is the $y$-extension of the fault. Using the method of (1-32) to (1-35), we use

$$
\left.\begin{array}{c}
r=r_{0} \vartheta \quad \vartheta^{2}=1-\frac{2}{r_{0}}\left(\xi \cos \theta_{0} \cos \delta+\eta \sin \theta_{0}\right)+\frac{\xi^{2} \cos ^{2} \delta+\eta^{2}}{r_{0}^{2}} \\
\frac{1}{2} r_{0}\left(1-\vartheta^{2}\right) \cong \xi \cos \theta_{0} \cos \delta+\eta \sin \theta_{0}
\end{array}\right\}
$$

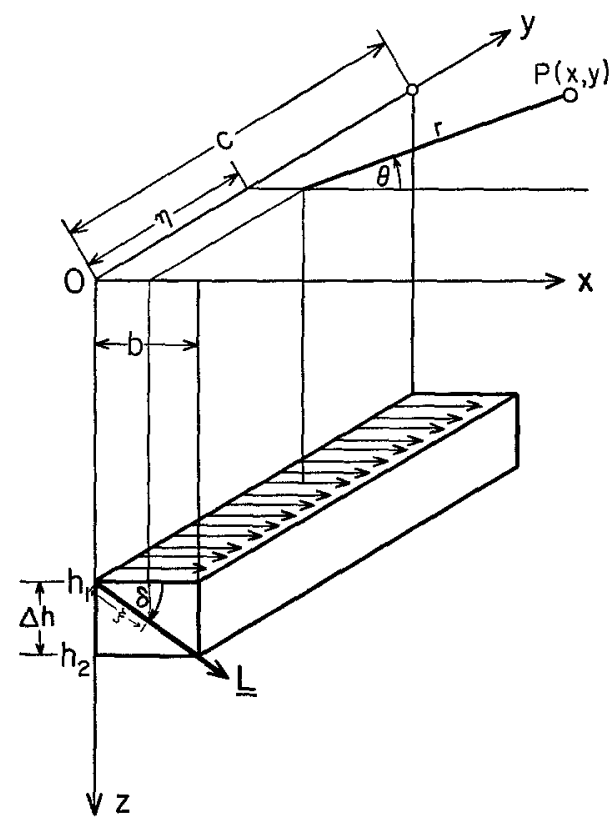

FIG. 6. Geometry of dip-slip fault with arbitrary dip angle.

The integration yields

$$
\begin{aligned}
\left(U_{r}^{R}\right)_{x} & =\frac{C_{1}^{\prime} L}{\mu} \frac{\cos \theta_{0}}{\sqrt{r_{0}}} \sqrt{K_{\beta}} \\
& \left\{\frac{\sin Q_{2}^{R}}{Q_{2}{ }^{R}} e^{-i Q_{1}{ }^{R}-K_{n} h_{1} d_{2}}-E_{1} \frac{\sin Q_{1}^{R}}{Q_{1}{ }^{R}} e^{-i Q_{2}{ }^{R}-K_{n} h_{1} d_{1}}\right\} \frac{\sin Z_{R}}{Z_{R}} e^{i(\tau R+(3 \pi / 4))} \\
\left(U_{\theta}{ }^{R}\right)_{x} & =\frac{C_{2}{ }^{\prime} L}{\mu} \frac{\sin \theta_{0}}{\mathrm{r}_{0}^{3 / 2}} \frac{1}{\sqrt{K_{\beta}}} \\
& \cdot\left\{\frac{\sin Q_{2}{ }^{R}}{Q_{2}{ }^{R}} e^{-i Q_{2}{ }^{R}-K_{n} h_{1} d_{2}}-E_{1} \frac{\sin Q_{1}{ }^{R}}{Q_{1}{ }^{R}} e^{-i Q_{1}{ }^{R}-K_{n} h_{1} d_{1}}\right\} \frac{\sin Z_{R}}{Z_{R}} e^{i\left(\tau_{R}-(3 \pi / 4)\right)} \\
\left(U_{z}{ }^{R}\right)_{x} & =\frac{C_{3}{ }^{\prime} L}{\mu} \frac{\cos \theta_{0}}{\sqrt{r_{0}}} \sqrt{K_{\beta}} \\
& \cdot\left\{\frac{\sin Q_{2}{ }^{R}}{Q_{2}{ }^{R}} e^{-i Q_{2}{ }^{R}-K_{n} h_{1} d_{2}}-E_{2} \frac{\sin Q_{1}^{R}}{Q_{1}{ }^{R}} e^{-i Q_{1}{ }^{R}-K_{n} h_{1} d_{1}}\right\} \frac{\sin Z_{R}}{Z_{R}} e^{i(\tau R+(\pi / 4))}
\end{aligned}
$$




$$
\begin{array}{r}
\left.Q_{1}{ }^{R}=\frac{\omega \bar{\sigma}}{2 C_{R}}\left\{\left(\frac{C_{R}}{v}-\cos \theta_{0} \cos \delta\right)+i \sin \delta \frac{\sqrt{\gamma^{2}-1}}{\gamma}\right\}\right) \\
\left.Q_{2}{ }^{R}=\frac{\omega \bar{\sigma}}{2 C_{R}}\left\{\left(\frac{C_{R}}{v}-\cos \theta_{0} \cos \delta\right)+i \sin \delta \frac{\sqrt{\gamma^{2}-\frac{1}{3}}}{\gamma}\right\}\right) \\
Z_{R}=\frac{\omega c \sin \theta_{0}}{2 C_{R}} ; \quad \tau_{R}=\omega\left(t-\frac{r_{0}}{C_{R}}\right)-Z_{R}
\end{array}
$$

In a similar manner we may obtain the suitable expressions for the contribution due to $L_{z}$. Combining them all, we have for the total Rayleigh-radiation of the dip-slip fault-plane:

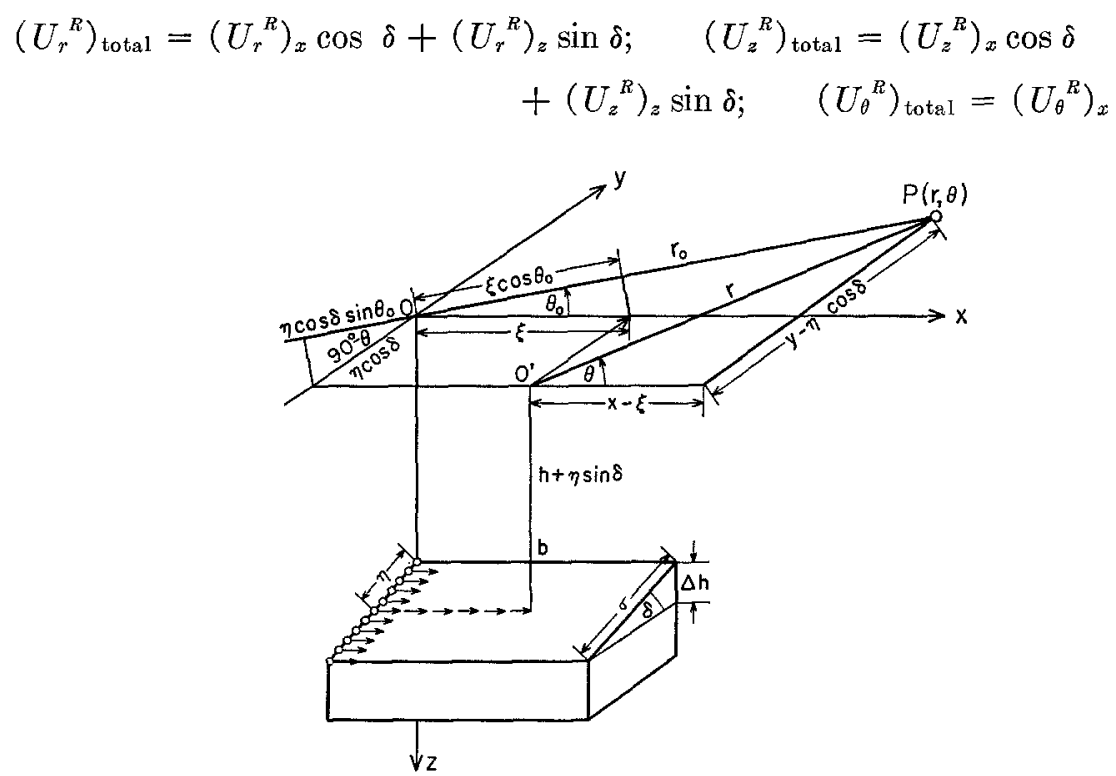

FIg. 7. Geometry of strike-slip fault with arbitrary dip angle.

\subsection{STRIKE-SLIP FAULT WITH ARBITRARY DIP ANGLE}

The situation is displayed in figure 7. Applying the former method we get, with

$$
\left.\begin{array}{c}
r=r_{0} \vartheta \quad r^{2}=(x-\xi)^{2}+(y-\eta \cos \delta)^{2} \\
\vartheta^{2}=1-\frac{2}{r_{0}}\left(\eta \sin \theta_{0} \cos \delta+\xi \cos \theta_{0}\right)+\frac{\xi^{2}+\eta^{2} \cos ^{2} \delta}{r_{0}^{2}}
\end{array}\right\}
$$

the approximation:

$$
\frac{1}{2} r_{0}\left(1-\vartheta^{2}\right) \cong \xi \cos \theta_{0}+\eta \sin \theta_{0} \cos \delta
$$

Using (1-32) and (1-22) to (1-24), and realizing the fault as described in figure 7, we obtain,

$$
U_{r}{ }^{R}=\frac{i C_{1} L}{\mu \sigma b} K_{\beta} \int_{0}^{b} \int_{0}^{\sigma}\left\{\begin{array}{l}
e^{-K_{n} d_{1}\left(h_{1}+\eta \eta \sin \delta\right)} \\
-E_{1} e^{-K_{n} d_{2}\left(h_{1}+\eta \sin \delta\right)}
\end{array}\right\} H_{0}^{(2)}\left(K_{n} r\right) e^{i \omega(t-(\xi / v))} \cos \theta d \eta d \xi
$$


Evaluating this double integral along the lines stated above, we get, to terms of the order of $1 / \sqrt{r_{0}}$ :

$$
\begin{aligned}
U_{r}^{R}=\frac{\left.L C_{1}^{\prime}\right]}{\mu} \sqrt{K_{\beta}} \frac{\cos \theta_{0}}{\sqrt{r_{0}}}\left\{\frac{\sin \mathrm{X}_{R}}{\mathrm{X}_{R}}\right\} \\
\cdot\left\{\frac{\sin \Lambda_{1}^{R}}{\Lambda_{1}^{R}} e^{-i \Lambda_{1} R-h_{1} K_{n} d_{1}}-E_{1} \frac{\sin \Lambda_{2}^{R}}{\Lambda_{2}^{R}} e^{-i \Lambda_{2} R-h_{1} K_{n} d_{2}}\right\} e^{i\left(\varphi_{R}+(3 \pi / 4)\right)}
\end{aligned}
$$

and similarly

$$
\begin{aligned}
U_{\theta}{ }^{R}=\frac{L C_{2}{ }^{\prime}}{\mu} \frac{1}{\sqrt{K_{\beta}}} \frac{\sin \theta_{0}}{r_{0}^{3 / 2}}\left\{\frac{\sin \mathrm{X}_{R}}{\mathrm{X}_{R}}\right\} \\
\cdot\left\{\frac{\sin \Lambda_{1}^{R}}{\Lambda_{1}^{R}} e^{-i \Lambda_{1} R-h_{1} K_{n} d_{1}}-E_{1} \frac{\sin \Lambda_{2}^{R}}{\Lambda_{2}^{R}} e^{-i \Lambda_{2} R-h_{1} K_{n} d_{2}}\right\} e^{i\left(\varphi_{R}-(3 \pi / 4)\right)} \\
U_{z}{ }^{R}=\frac{L C_{3}^{\prime}}{\mu} \sqrt{K_{\beta}} \frac{\cos \theta_{0}}{\sqrt{r_{0}}}\left\{\frac{\sin \mathrm{X}_{K}}{\mathrm{X}_{R}}\right\} \\
\cdot\left\{\frac{\sin \Lambda_{1}^{R}}{\Lambda_{1}^{R}} e^{-i \Lambda_{1} R-h_{1} K_{n} d_{1}}-E_{2} \frac{\sin \Lambda_{2}^{R}}{\Lambda_{2}^{R}} e^{-i \Lambda_{2}{ }^{R}-h_{1} K_{n} d_{2}}\right\} e^{i\left(\left(\varphi_{R}+(\pi / 4)\right)\right.}
\end{aligned}
$$

with

$$
\left.\begin{array}{l}
\Lambda_{1}{ }^{R}=\frac{\omega \sigma}{2 C_{R}}\left(\sin \theta_{0} \cos \delta+i \frac{\sqrt{\gamma^{2}-1}}{\gamma} \sin \delta\right) \\
\Lambda_{2}{ }^{R}=\frac{\omega \sigma}{2 C_{R}}\left(\sin \theta_{0} \cos \delta+i \frac{\sqrt{\gamma^{2}-\frac{1}{3}}}{\gamma} \sin \delta\right)
\end{array}\right\}
$$

Here again $\Lambda_{1}$ and $\Lambda_{2}$ are complex. This is so because the depth does not have a phase effect while the extension along the $y$-axis, does. The former results are easily generalized for a radiating couple: we differentiate each of (1-37) to (1-39) with respect to $y$, with $\partial / \partial y=\sin \theta_{0}\left(\partial / \partial r_{0}\right)+\left(\cos \theta_{0} / r_{0}\right)\left(\partial / \partial \theta_{0}\right)$. Neglecting terms higher than $1 / \sqrt{r_{0}}$ we find that the only terms which contribute (to this order of magnitude) are those which arise from differentiating the exponent $e^{i \varphi_{R}}$. It means that in order to obtain the couple solutions we have to multiply (1-37) to (1-39) by the factor $K_{n} \sin \theta_{0} e^{-(\pi i / 2)}$ :

$$
\begin{aligned}
& \left(U_{r}{ }^{R}\right)_{\text {couple }}=\frac{\sin 2 \theta_{0}}{\sqrt{r_{0}}} g_{r}(\omega) \sqrt{K_{\beta}}\left\{\frac{\sin \mathrm{X}_{R}}{\mathrm{X}_{R}}\right\} e^{i\left(\varphi_{R}-(3 \pi / 4)\right)}+0\left\{\left(K_{n} r_{0}\right)^{-3 / 2}\right\} \\
& \left.\left(U_{\theta}{ }^{R}\right)_{\text {couple }}=\frac{\sin ^{2} \theta_{0}}{r_{0}^{3 / 2}} g_{\theta}(\omega) \frac{1}{\sqrt{K_{\beta}}}\left\{\frac{\sin \mathrm{X}_{R}}{\mathrm{X}_{R}}\right\} e^{i\left(\varphi_{R}-(\pi / 4)\right)}+0\left\{K_{n} r_{0}\right)^{-5 / 2}\right\} \\
& \left(U_{z}{ }^{R}\right)_{\text {couple }}=\frac{\sin 2 \theta_{0}}{\sqrt{r_{0}}} g_{z}(\omega) \sqrt{K_{\beta}}\left\{\frac{\sin \mathrm{X}_{R}}{\mathrm{X}_{R}}\right\} e^{i\left(\varphi_{R}+(3 \pi / 4)\right)}+0\left\{\left(K_{n} r_{0}\right)^{-3 / 2}\right\}
\end{aligned}
$$

If one wishes, one may obtain also solutions for bidirectional fault by superposing on $\left(1-37^{\mathrm{a}}\right)$ to $\left(1-39^{\mathrm{a}}\right)$ the solution $-U_{i}\left(\pi-\theta_{0}\right)$. (Motion starts simultaneously from the source in two opposite directions.) The same applies to (1-53) to (1-54) and (1-73) to (1-75). 


\subsection{A WORKED EXAMPLE}

Let us now investigate in detail the case of the strike-slip fault-plane, for a concrete example, in order to see the behaviour of the various parameters which take part in the mathematical formulation of the resulting displacements. The limitations on the choice of the parameters is given by (1-35) and (1-36). The condition $1 \ll\left(\omega r_{0} / C_{R}\right)$ has obviously the physical meaning:

$$
r_{0} \gg \frac{\lambda}{2 \pi}
$$

Looking back on the previous equations we note that the ratio $b / \lambda$ appears in the parameters, $X$ and $Y$ and has a dominating influence on the function $\{\sin \mathrm{X} / \mathrm{X}$ ?

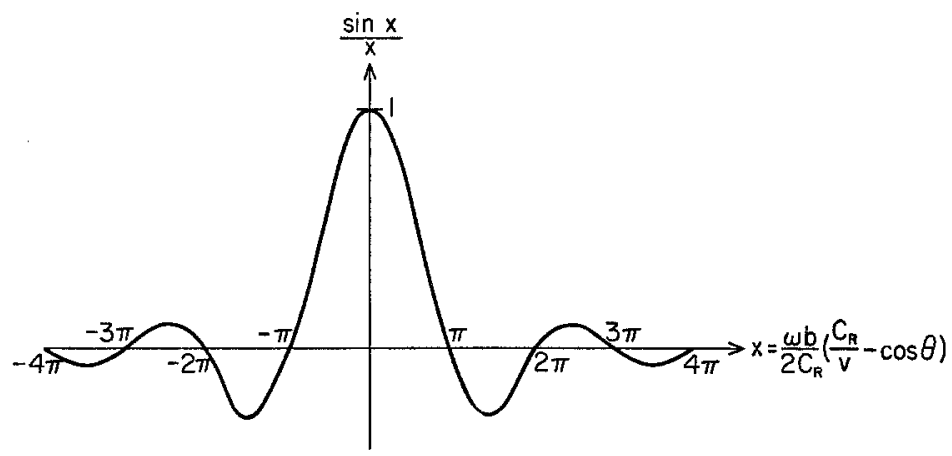

Frg. 8. Finiteness factor as function of azimuthal angle and source parameters.

Let us investigate the radial Rayleigh-displacement for the case $\delta=\pi / 2$ with a couple-type moving source. Multiplying (1-37) by $\omega / C_{R} \sin \theta_{0} e^{(\pi i / 2)}$ we have:

$$
\begin{aligned}
\left|U_{r}^{R}\right|=\mid \frac{L C_{5}^{\prime}}{\mu \cdot \Delta h} \frac{\sin 2 \theta_{0}}{\sqrt{T r_{0}}} & \\
& \cdot\left\{\left(e^{-\left(a_{1} / T\right)}-e^{-\left(a_{2} / T\right)}\right)-\Upsilon_{1}\left(e^{-\left(a_{3} / T\right)}-e^{-\left(a_{4} / T\right)}\right\} \frac{\sin \mathrm{X}_{R}}{\mathrm{X}_{R}} \mid\right.
\end{aligned}
$$

with

$$
\begin{array}{ll}
a_{1}=\frac{2 \pi h_{1} \sqrt{\gamma^{2}-1}}{\gamma C_{R}} & a_{2}=\frac{2 \pi h_{2} \sqrt{\gamma^{2}-1}}{\gamma C_{R}} \\
a_{3}=\frac{2 \pi h_{1} \sqrt{\gamma^{2}-\frac{1}{3}}}{\gamma C_{R}} & a_{4}=\frac{2 \pi h_{2} \sqrt{\gamma^{2}-\frac{1}{3}}}{\gamma C_{R}}
\end{array}
$$

We shall now omit the phase factor, taking only the absolute value of the displacements. The amplitude factor of $\left|U_{r}^{R}\right|$ is built of three sub-factors. The factor $\sin 2 \theta_{0}$ is characteristic for the dipole field of a point source. The factor containing the four exponentials plays the role of an attenuator and has a single zero for some $T$ which depends on the constants $\alpha_{j}$ and $\Upsilon_{1}$. ( $U_{z}$ also has such a zero.) The third factor, of the type $(\sin X / X)$, is a contribution of the finiteness of the source in the direction of the propagating disturbance. It is this factor which is responsible for 
the deviation from the pure dipole-field. This function has its zeros at

$$
\frac{\omega b}{2 C_{R}}\left(\frac{C_{R}}{v}-\cos \theta_{0}\right)=n \pi, \quad n=1,2, \cdots
$$

We rewrite this relation in the form

$$
\frac{b}{v} C_{R}-b \cos \theta_{0}=n \lambda
$$

from which the physical significance is clear: it is the condition for destructive interference at the point of observation. This case is analogous in many ways to Frauenhoffer diffraction through a rectangular slit and should not be mistaken for the condition of constructive interference in the case of two separated point-sources. The important thing to remember is that as long as

$$
n \pi<\left|\frac{\omega b}{2 C_{R}}\left(\frac{C_{R}}{v}-\cos \theta_{0}\right)\right|<(n+1) \pi,
$$

no additional zeros are introduced into the normal couple pattern, and no more than a relative reduction in amplitude is expected. Thus, a bound for $b$ can be set if the observed pattern for a certain frequency does not deviate from the pointcouple scheme. Furthermore, the excessive number of zeros in any quadrant will be equal to the greatest integer in $[2(b / \lambda)]$ with an uncertainty of unity. The number of lobes is therefore a clear measure for the dimensions of the source. Another interesting outcome of the dependence on $(\sin \mathrm{X}) / \mathrm{X}$ is exhibited by the ratio of amplitudes at equal distances in two opposite directions:

$$
\left|\frac{U_{r}^{R}\left(\theta_{0}\right)}{U_{r}^{R}\left(\pi+\theta_{0}\right)}\right|=\left|\frac{\frac{\sin \frac{\pi b}{\lambda}\left(\frac{C_{R}}{v}-\cos \theta_{0}\right)}{\frac{\pi b}{\lambda}\left(\frac{C_{R}}{v}-\cos \theta_{0}\right)}}{\frac{\sin \frac{\pi b}{\lambda}\left(\frac{C_{R}}{v}+\cos \theta_{0}\right)}{\frac{\pi b}{\lambda}\left(\frac{C_{R}}{v}+\cos \theta_{0}\right)}}\right|
$$

It is important to note that this ratio, at least for the strike-slip fault, is independent of the source time-function as well as of the layering of the elastic halfspace. We shall later make use of this fact. It is obvious from (1-81) that this ratio may acquire any value from zero to infinity. The phenomenon that surface-waves amplitudes show a very strong dependence on the azimuth of the station has already been established by Gutenberg and Richter (1934, 1936), Benioff (1955) and Gutenberg (1955). This ratio approaches unity as $(b / \lambda)$ decreases, and is another measure for the source extension, and the speed of rupture.

In our computation, the following magnitudes have been used: $b=60 \mathrm{~km}$, $h_{1}=15 \mathrm{~km}, h_{2}=20 \mathrm{~km}(\Delta h=5 \mathrm{~km}), \pi b / C_{R}=50$, so that $a_{1}=9$ sec, $a_{2}=12$ sec, $a_{3}=18$ sec, and $a_{4}=24$ sec. With these numbers, we see that the function $\left(e^{-(9 / T)}-e^{-(12 / T)}\right)-0.80\left(e^{-(18 / T)}-e^{-(24 / T)}\right)$ has a zero for $T \cong 20$ sec. It is positive for $T<20$ sec and negative for $T>20$ sec. Results are given in figures $9-20$. 
The first set (figs. 9-14) gives the dependence of $U_{r}$ on $\theta_{0} . \theta_{0}$ is given at intervals of $5^{\circ}$ and the parameter $C_{R} / v$ runs over the set: $C_{R} / v=4(v \cong 1(\mathrm{~km} / \mathrm{sec})), 0.9194$ $(v \cong \beta), 0.5308(v \cong \alpha)$. Periods have been chosen as $T=15 \mathrm{sec}, 25 \mathrm{sec}, 50 \mathrm{sec}$. For periods higher than $50 \mathrm{sec}$ the deviation from the couple field is small. The second set of figures (figs. 15-17) gives $\left|U_{r}\right|$ as a function of $T$, where $\theta_{0}$ is the parameter, and in the third group (figs. 18-20) the amplitude is given as a function of the ratio $C_{R} / v$, with $\theta_{0}$ as a parameter.
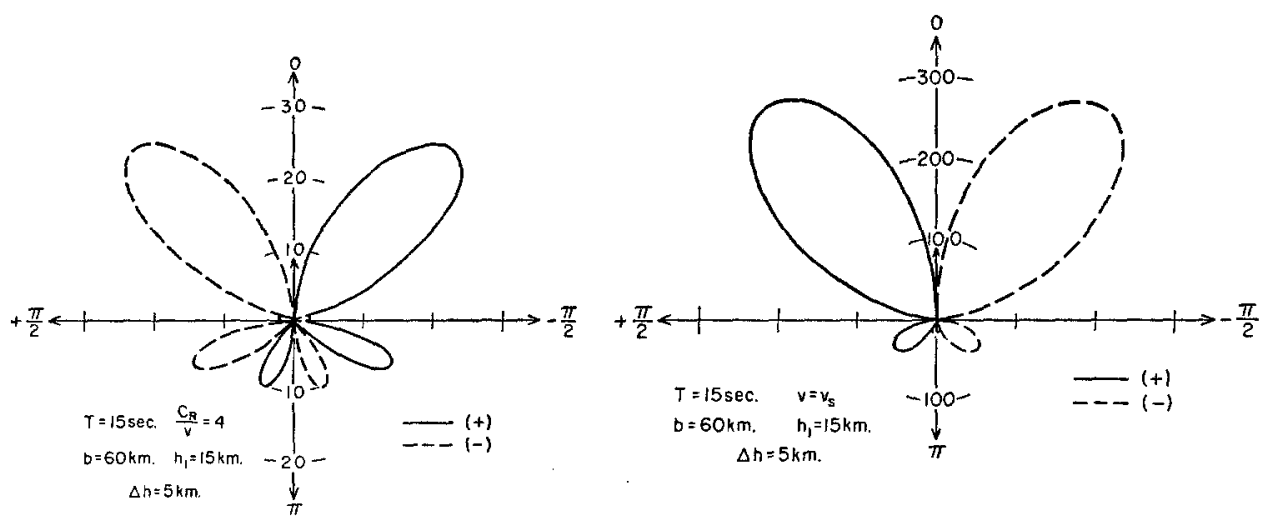

FIG. 9. Azimuthal distribution of radial displacements for a vertical strike-slip fault.

FIG. 10. Azimuthal distribution of radial displacements for a vertical strike-slip fault.

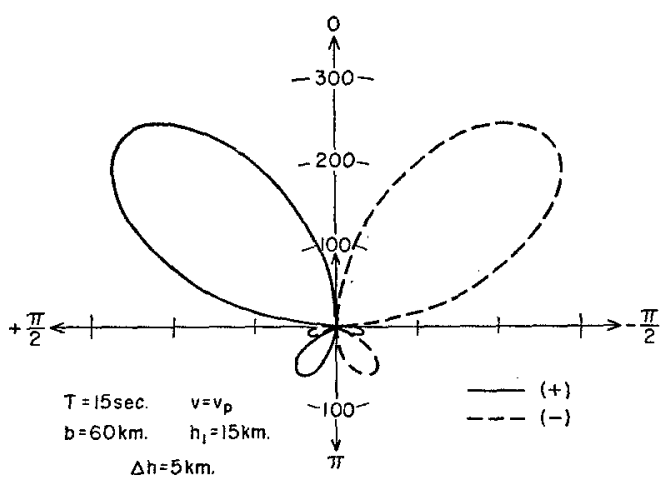

FIG. 11. Azimuthal distribution of radial displacements for a vertical strike-slip fault.

FIG. 12. Azimuthal distribution of radial displacements for a vertical strike-slip fault.

To see what happens for dip angles other than $\delta=\pi / 2$ we shall take the other extremity, $\delta=0$. The amplitude factor of $U_{r}$ for this case is, from (1-73):

$$
\left|U_{r}^{R}\right|=\left|\frac{C_{6}^{\prime} L}{\mu} \frac{\sin 2 \theta_{0}}{\sqrt{r_{0}}} \frac{1}{T^{3 / 2}}\left\{e^{-\left(a_{1} / T\right)}-E_{1} e^{-\left(a_{3} / T\right)}\right\}\left\{\frac{\sin X_{R}}{X_{R}}\right\} \frac{\sin \left(\frac{\pi \sigma}{\lambda} \sin \theta_{0}\right)}{\left(\frac{\pi \sigma}{\lambda} \sin \theta_{0}\right)}\right|
$$

where $a_{1}, a_{3}$ are the same as in (1-79), and $E_{1}=2 \gamma^{2} /\left(2 \gamma^{2}-1\right)$. Examining (1-82) we notice that for the previous values of $\sigma=5 \mathrm{~km}$ and $T \geqq 15 \mathrm{sec}$, we always 
have $\pi \sigma / \lambda \sin \theta_{0} \leqq \frac{1}{3} \sin \theta_{0}<1$, i.e., the deviation from the results for $\delta=\pi / 2$ will be small unless $\sigma$ acquires higher values. We conclude therefore, that the dip

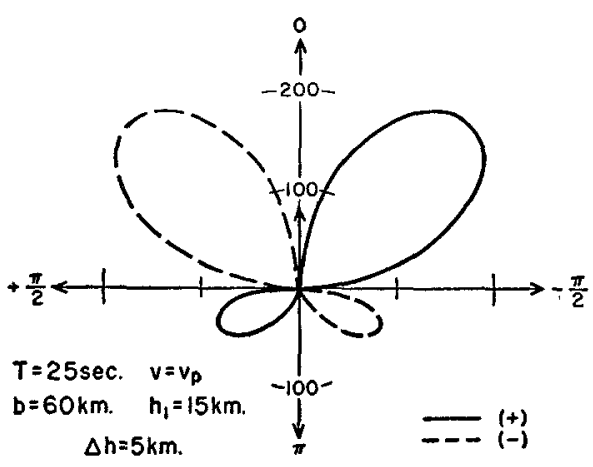

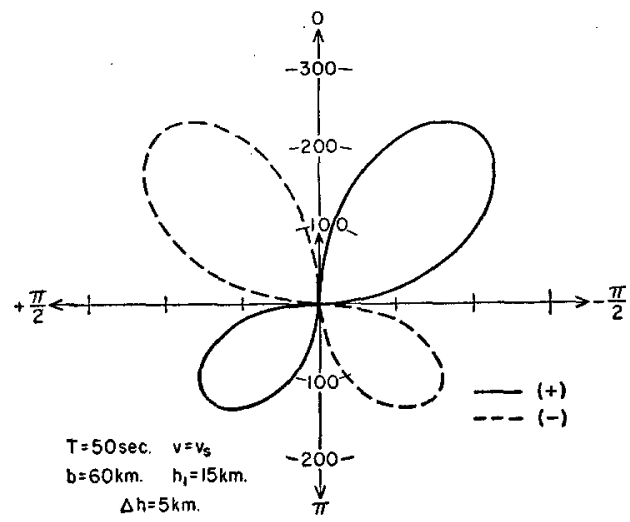

Fig. 13. Azimuthal distribution of radial displacements for a vertical strike-slip fault. FIG. 14. Azimuthal distribution of radial displacements for a vertical strike-slip fault.

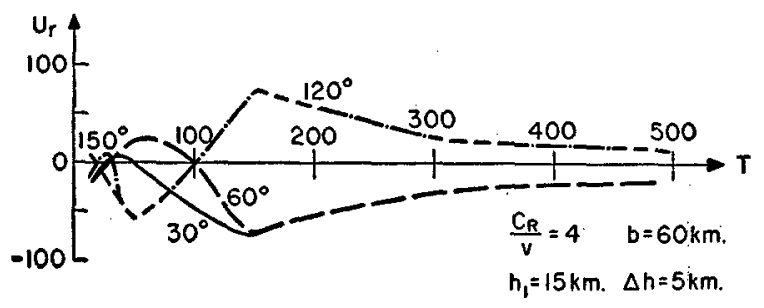

Fig. 15

FIG. 15. Spectral distribution of radial displacements for a vertical strike-slip fault.
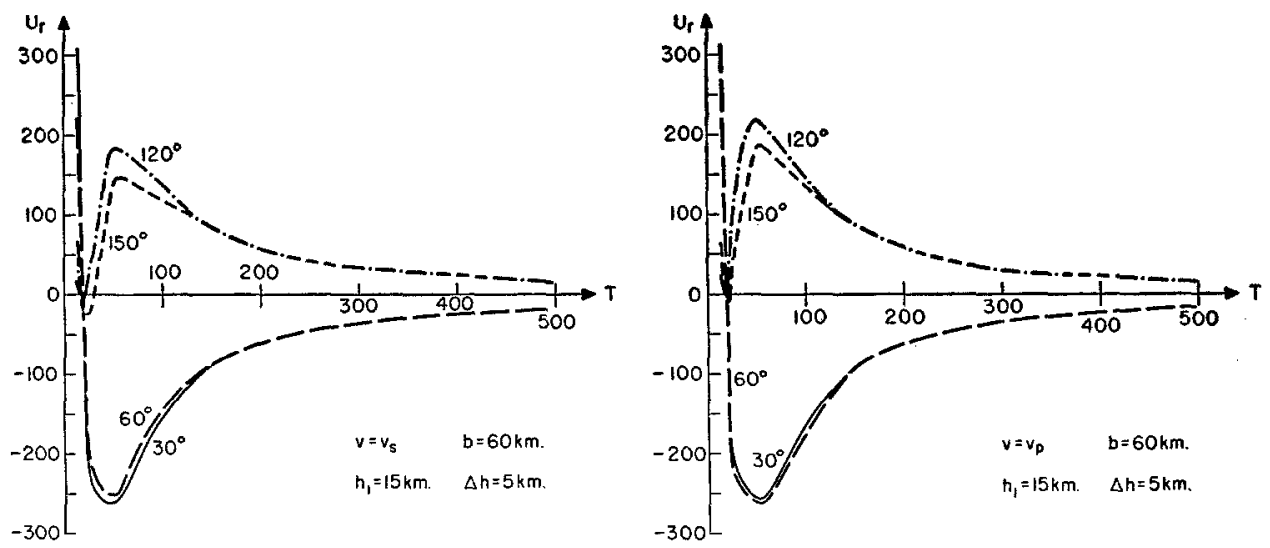

FIG. 16. Spectral distribution of radial displacements for a vertical strike-slip fault.

FIG. 17. Spectral distribution of radial displacements for a vertical strike-slip fault.

angle for the strike-slip case with $\sigma \ll b$ has no fundamental influence on the wave pattern. The computation for $U_{z}{ }^{R}$ may be carried along the same lines given hitherto, and there is essentially no remarkable qualitative difference of the wave pattern for this case. 


\subsection{GENERALIZATION TO A LAYERED HALF-SPACE}

The former results may be generalized to an inhomogeneous elastic medium which in turn may be represented by a multi-layered half-space. Consider a horizontal simple-force placed in the jth layer of an $m$-layered half-space, at depth $h$ below the free surface. We shall follow the method of analysis given in section 1.2: After defining $3 m+6$ scalar potential-functions there will be $6 m+9$ boundary-condi-
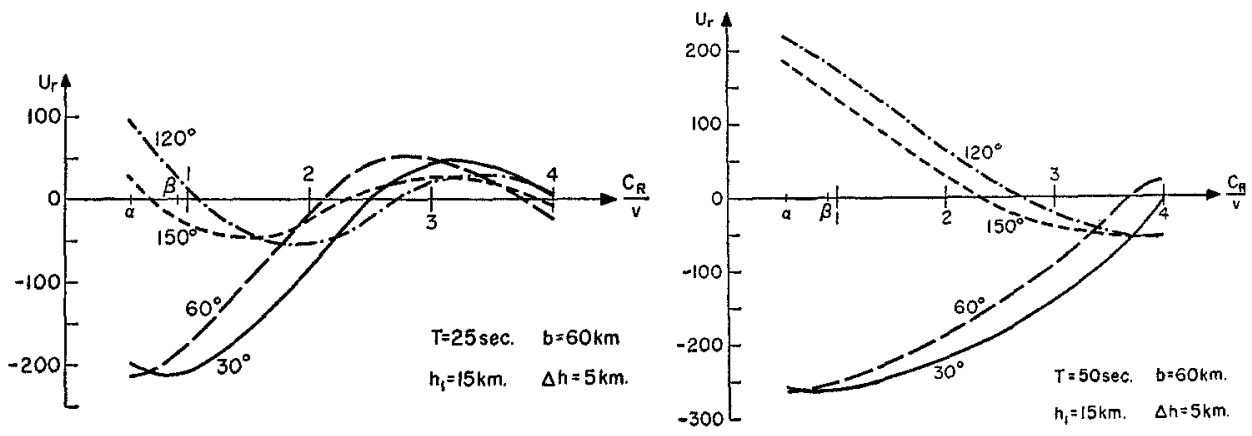

FIG. 18. Radial displacements for the vertical strike-slip fault, as a function of the velocity ratio $C_{R} / v$.

FIG. 19. Radial displacement for the vertical strike-slip fault, as a function of the velocity ratio $C_{R} / v$.

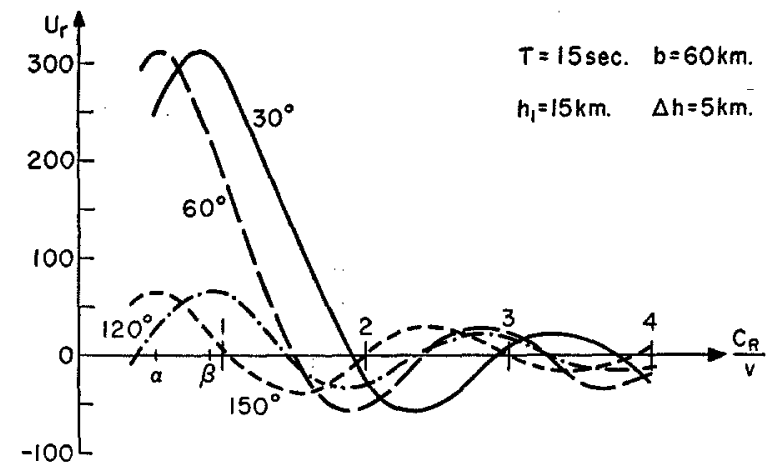

FIG. 20. Radial displacements for the vertical strike-slip fault, as a function of the velocity ratio $C_{R} / v$.

tion equations for the $6 m+9$ indeterminate coefficients. The surface Rayleighdisplacements will have the form:

$$
\begin{aligned}
& u_{r}=\frac{i L}{2 \mu_{j}} \cos \theta_{0} e^{i \omega t} \sum_{n=1} K_{n} \frac{{ }_{m} F_{1}{ }^{j}\left(K_{n}\right)}{\frac{\partial_{m} F_{0}\left(K_{n}\right)}{\partial K_{n}}}\left\{H_{0}^{(2)}\left(K_{n} r\right)-\frac{1}{K_{n} r} H_{1}^{(2)}\left(K_{n} r\right)\right\} \\
& u_{\theta}=\frac{i L}{2 \mu_{j} r} \sin \theta_{0} e^{i \omega t} \sum_{n=1} \frac{{ }_{m} F_{1}{ }^{j}\left(K_{n}\right)}{\frac{\partial_{m} F_{0}\left(K_{n}\right)}{\partial K_{n}}} H_{1}^{(2)}\left(K_{n} r\right) \\
& u_{z}=\frac{i L}{2 \mu_{j}} \cos \theta_{0} e^{i \omega t} \sum_{n=1} K_{n} \frac{{ }_{m} F_{2}{ }^{j}\left(K_{n}\right)}{\frac{\partial_{m} F_{0}\left(K_{n}\right)}{\partial K_{n}}} H_{1}^{(2)}\left(K_{n} r\right) \quad m=0,1,2 \cdots
\end{aligned}
$$


Here ${ }_{m} F_{0}\left(K_{n}\right)$ stands for the system determinant for the layered half-space and ${ }_{m} F_{1}{ }^{j}\left(K_{n}\right),{ }_{m} F_{2}{ }^{j}\left(K_{n}\right)$ are some linear combinations of the cofactors of the system determinant. These expressions are functions of the source depth, the width of the layers and the elastic parameters of these layers. The summation extends over all the possible modes.

The integrated Rayleigh-pattern for a vertical strike-slip fault with a coupletype source is given later in (3-3) and (3-4). To obtain the exact analytical form of $g_{r}{ }^{m}$ and $g_{z}{ }^{m}$ (defined there) in case the fault extends downward through $m_{0}$ layers, one has to evaluate ${ }_{m} F_{1}{ }^{j}\left(K_{n}\right)$ and ${ }_{m} F_{2}{ }^{j}\left(K_{n}\right)$ for $m_{0}$ different cases. Each case corresponding to the source being located at a different layer. The resulting $2 m_{0}$ functions ${ }_{m} F_{1}{ }^{j}(h, \omega),{ }_{m} F_{2}{ }^{j}(h, \omega), j=1,2, \cdots m_{0} \leqq m$ are then integrated individually with respect to $h$ between the proper limits. Finally we have:

$$
\begin{aligned}
& g_{r}{ }^{m}=w_{1} \frac{\sum_{j=1}^{m_{0}} \int_{H j-1}^{H j}{ }_{m} F_{1}^{j}(\omega, h) d h}{\frac{\partial_{m} F_{0}\left(K_{n}\right)}{\partial K_{n}}} \quad(n=1) \\
& g_{z}{ }^{m}=w_{2} \frac{\sum_{j=1}^{m_{0}} \int_{H j-1}^{H j}{ }_{m} F_{2}^{j}(\omega, h) d h}{\frac{\partial_{m} F_{0}\left(K_{n}\right)}{\partial K_{n}}}, \quad m=0,1,2, \cdots
\end{aligned}
$$

$H_{j}$ is the width of the $j$ th layer. $H_{0}=0 . w_{1}$ and $w_{2}$ are positive real constants. It has been assumed here that the fault extends from the free surface. Expressions for ${ }_{1} F_{1}{ }^{1}(K n),{ }_{1} F_{2}{ }^{1}(K n)$ and ${ }_{1} F_{0}(K n)$ are given by Yanovskaya (1958). It can be shown that the surface displacements are specified already by $6 m+3$ indeterminate coefficients. That is so because the corresponding differences between the 6 coefficients above the source and the 6 coefficients below the source lead to terms of zero residue in the integral representation of the surface displacements. It has also been proved by Keylis-Borok (references given in Yanovskaya 1958) that $\sum_{m}$, the $(6 m+3)$ by $(6 m+3)$ determinant of the total system, is factorable into a Rayleigh determinant ${ }_{m} F_{0}$ of order $3 m+3$ and a Love determinant $\Delta_{L}$ of order $3 m$ :

$$
\sum_{m}=\left\{-K^{2 m+1}\left(K_{\beta}\right)_{\text {half space }} \prod_{j=1}^{m} K_{\beta m}^{2}\right\}_{m} F_{0} \cdot \Delta_{L}
$$

where $K_{\beta m}$ is the shear wave-number of the $m$ th layer.

\section{Chapter 2-Love Waves from Finite Moving Sources}

\subsection{INTRODUCTION}

The effect of the finiteness of the seismic focus on the Love-wave radiation pattern is investigated. We start from the exact solution for the surface displacements due to a harmonic horizontal point-source, situated in a homogeneous layer overlaying a homogeneous half-space. A fault plane is then realized by moving this source along a line with finite speed and integrating the Love-poles contribution across a finite rectangle in the layer. Displacements are evaluated for long ranges and expressions are obtained for strike-slip and dip-slip fault types. Attention is 
focused mainly on a couple-type motion of a vertical strike-slip model. The theory is then applied to the $G$-wave pattern for which the results are also transformed into the time domain. It is also demonstrated how this theory may lead to an estimate of source parameters such as fault-length and rupture-velocity from phases and amplitudes ratio of $G$ or $R$ waves recorded at a single station.

All present computations and results will be limited to the first mode only, as higher modes are not observed under common circumstances. Consequently, $C_{L}(\omega)$ will stand for the first branch of the dispersion curve.

\subsection{THE HORIZONTAL-SOURCE SOLUTION}

The surface displacements for a simple horizontal force, acting at depth $h$ below the free surface in a layer of depth $H$ was given by Yanovskaya (1958). Using the

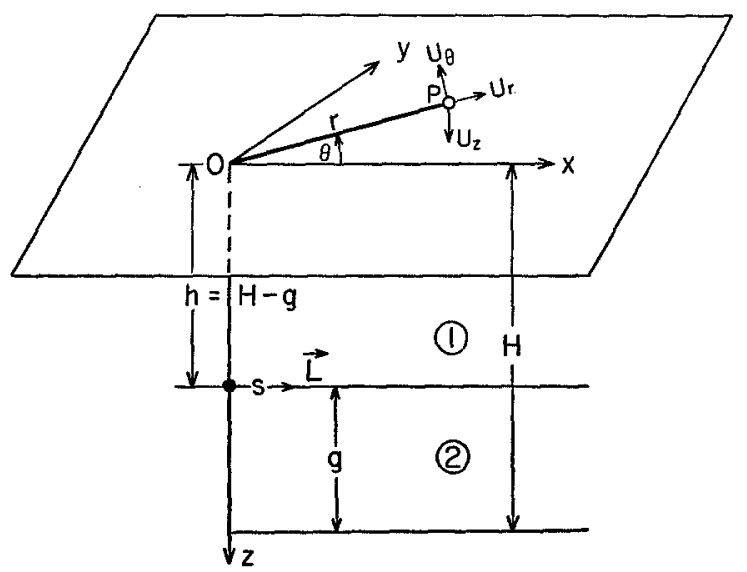

FIG. 21. Point source in a layer over a half-space.

method outlined in Chapter 1 for a horizontal force in the sense of section 1.2, located at $z=h<H$ (fig. 21), we have:

$$
\begin{aligned}
& u_{r}=-\frac{i L}{2 \mu_{1}} \frac{\cos \theta}{r} e^{i \omega t} H_{1}^{(2)}\left({ }_{n} K r\right) \frac{D_{L}\left({ }_{n} K, H, h\right)}{\left\{\frac{\partial \Delta_{L}\left({ }_{n} K, H\right)}{\partial_{n} K}\right\}} \\
& u_{\theta}=-\frac{i L}{2 \mu_{1}} \sin \theta e^{i \omega t}\left[H_{0}^{(2)}\left({ }_{n} K r\right)-\frac{1}{{ }_{n} K r} H_{1}^{(2)}\left({ }_{n} K r\right)\right] \frac{{ }_{n} K D_{L}\left({ }_{n} K, H, h\right)}{\left\{\frac{\partial \Delta_{L}\left({ }_{n} K, H\right)}{\partial_{n} K}\right\}} \\
& u_{z}=0,
\end{aligned}
$$

The suitable expressions for the cartesian components are accordingly:

$$
u_{x}=\frac{i L}{2 \mu_{1}} \sin ^{2} \theta e^{i \omega t} H_{0}^{(2)}\left({ }_{n} K r\right) \frac{{ }_{n} K D_{L}\left({ }_{n} K, H, h\right)}{\left\{\frac{\partial \Delta_{L}\left({ }_{n} K, H\right)}{\partial_{n} K}\right\}}+0\left[\left({ }_{n} K r\right)^{-3 / 2}\right]
$$




$$
\begin{aligned}
& u_{y}=-\frac{i L}{4 \mu_{1}} \sin 2 \theta e^{i \omega t} H_{0}^{(2)}\left({ }_{n} K r\right) \frac{{ }_{n} K D_{L}\left({ }_{n} K, H, h\right)}{\left\{\frac{\partial \Delta_{L}\left({ }_{n} K, H\right)}{\partial_{n} K}\right\}}+0\left[\left({ }_{n} K r\right)^{-3 / 2}\right] \\
& u_{z}=0 \text {, } \\
& n=1
\end{aligned}
$$

The notation incorporated in $(2-1)$ to $(2-6)$ is

$$
\left.\begin{array}{rlrl}
D_{L} & =\left\{\zeta \frac{\nu_{2}}{\nu_{1}} \operatorname{sh}\left(\nu_{1} H\right)+\operatorname{ch}\left(\nu_{1} H\right)\right\} \operatorname{ch}\left(\nu_{1} h\right) \\
\Delta_{L} & =\zeta \nu_{2} \operatorname{ch}\left(\nu_{1} H\right)+\nu_{1} \operatorname{sh}\left(\nu_{1} H\right) \\
\zeta & =\frac{\mu_{2}}{\mu_{1}} & \nu_{1} & =\sqrt{{ }_{n} K^{2}-K_{1}^{2}}=i \omega \alpha_{1} \\
h & =H-g & \nu_{2} & =\sqrt{{ }_{n} K^{2}-K_{2}^{2}}=\omega \alpha_{2} \\
K_{1} & =\frac{\omega}{\beta_{1}} & \alpha_{1} & =\sqrt{\frac{1}{\beta_{1}^{2}}-\frac{1}{C_{L}{ }^{2}}} \\
K_{2} & =\frac{\omega}{\beta_{2}} & \alpha_{2} & =\sqrt{\frac{1}{C_{L}{ }^{2}}-\frac{1}{\beta_{2}^{2}}} \\
{ }_{n} K & =\frac{\omega}{C_{L}} & \beta_{2} \geqq C_{L} \geqq \beta_{1}
\end{array}\right\}
$$

and $C_{L}=C_{L}(\omega)$ is given implicitly by the period equation

$$
\Delta_{L}=0
$$

Performing the operations indicated in (2-1) and (2-2) we get, with the aid of $(2-7)$ to $(2-10)$, for the leading term of $u_{\theta}$ : (we shall give henceforth the results for $u_{\theta}$ only, as $u_{r}$ varies like $r_{0}^{-3 / 2}$ ):

$$
u_{0}=-\frac{i L}{2 \mu_{1}} \sin \theta H_{0}^{(2)}\left({ }_{n} K r\right) e^{i \omega t} N_{0}(\omega) \cos \left(h \omega \alpha_{1}\right)+0\left[\left({ }_{n} K r\right)^{-3 / 2}\right]
$$

with

$$
N_{0}(\omega)=\frac{\alpha_{2} \omega}{\alpha_{2} \omega H+\zeta\left\{\frac{1+\left(\frac{\alpha_{2}}{\alpha_{1}}\right)^{2}}{1+\zeta^{2}\left(\frac{\alpha_{2}}{\alpha_{1}}\right)^{2}}\right\}}
$$

2.3 A MODEL FOR A DIP-SLIP FAULT IN THE LAYER

Imagine a fault plane striking in the direction of the $y$-axis and dipping at an angle $\delta$ with respect to the free surface (fig. 22). Assume further that the impulse starts at some depth $h_{1}$, in the layer, along a line $y=0$ to $y=c$, directed downwards, at the angle $\delta$. We may split the force $\vec{L}$ into a vertical component and a horizontal component: $L_{x}=|L| \cos \delta L_{z}=|L| \sin \delta$. The only contribution to Love waves 
arises from the $L_{x}$ component. Using the same method as given in section 1.6 we get for the integrated radiation:

$$
\begin{aligned}
& U_{\theta}{ }^{L}=\frac{L}{2 \mu_{1} \sqrt{2 \pi}} \frac{\sin \theta_{0} \cos \delta}{\sqrt{n} \bar{K} r_{0}} N_{0}(\omega) \frac{\sin Z_{L}}{Z_{L}} \\
& \cdot\left\{\frac{\sin Q_{1}{ }^{L}}{Q_{1}{ }^{L}} e^{i\left(\omega h_{1} \alpha_{1}+Q_{1} L\right)}+\frac{\sin Q_{2}{ }^{L}}{Q_{2}{ }^{L}} e^{-i\left(\omega h_{1} \alpha_{1}-Q_{2} L\right)}\right\} e^{\left.i\left(T_{L}+3 \pi / 4\right)\right)}
\end{aligned}
$$

with

$$
Z_{L}=\frac{\omega c}{2 c_{L}} \sin \theta_{0} \quad \tau_{L}=\omega\left(t-\frac{r_{0}}{C_{L}}\right)-Z_{L}
$$

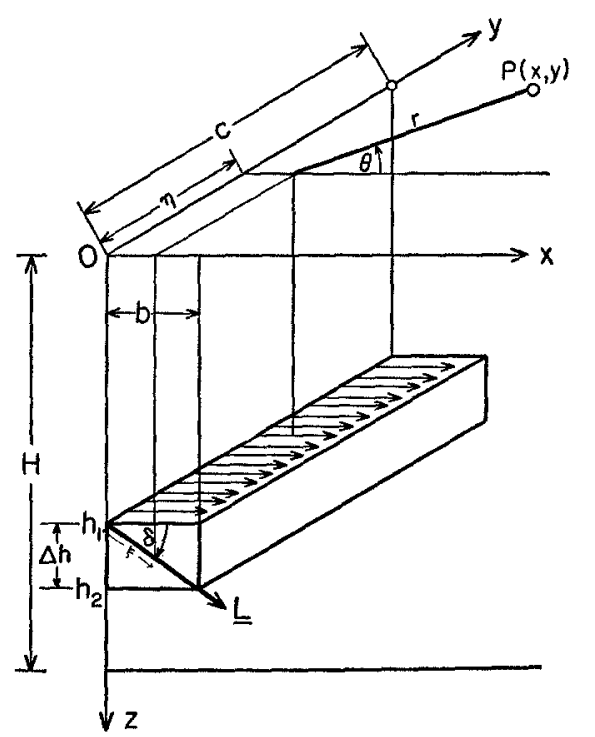

FIG. 22. Geometry of the dip-slip fault inside the layer.

$$
\begin{aligned}
Q_{1}{ }^{L} & =\frac{\omega \bar{\sigma}}{2 c_{L}}\left\{\left(\frac{C_{L}}{v}-\cos \theta_{0} \cos \delta\right)+\alpha_{1} C_{L} \sin \delta\right\} \\
Q_{2}{ }^{L} & =\frac{\omega \bar{\sigma}}{2 c_{L}}\left\{\left(\frac{C_{L}}{v}-\cos \theta_{0} \cos \delta\right)-\alpha_{1} C_{L} \sin \delta\right\}
\end{aligned}
$$

under the conditions

$$
\left|\frac{\omega r_{0}}{c_{L}}\right| \gg 1, \quad\left(\frac{\bar{\sigma}}{r_{0}}\right) \ll 1 \quad\left(\frac{c}{r_{0}}\right) \ll 1
$$

\subsection{A MODEL FOR A STRIKE-SLIP FAULT IN THE LAYER}

The geometrical configuration of the fault in this case is clear from figure 23 . The integrated effect of the fault is given by the double integral:

$$
\begin{aligned}
U_{\theta}{ }^{L}=-\frac{i L}{2 \mu_{1}} \sin \theta_{0} N_{0}(\omega) \int_{0}^{b} \int_{0}^{\sigma} H_{0}^{(2)}\left(K_{n} r\right) \\
\cdot \cos \left[\omega \alpha_{1}\left(h_{1}+\eta \sin \delta\right)\right] e^{i \omega(i-(\xi / v))} d \eta d \xi
\end{aligned}
$$


which yields

$$
\begin{aligned}
U_{\theta}{ }^{L}=\sqrt{\frac{2}{\pi}} \frac{L}{4 \mu_{1}} \frac{\sin \theta_{0}}{\sqrt{r_{0}}} N_{0}(\omega) & \frac{1}{\sqrt{n K}}\left\{\frac{\sin \Lambda_{1}{ }^{L}}{\Lambda_{1}{ }^{L}} e^{i\left(\omega \alpha_{1} h_{1}+\Lambda_{1} L\right)}\right. \\
& \left.+\frac{\sin \Lambda_{2}{ }^{L}}{\Lambda_{2}{ }^{L}} e^{-i\left(\omega \alpha_{1} h_{1}-\Lambda_{2} L\right.}\right\} \frac{\sin \mathrm{X}_{L}}{\mathrm{X}_{L}} e^{i\left(\varphi_{L}+(3 \pi / 4)\right)}
\end{aligned}
$$

with

$$
\varphi_{L}=\omega\left(t-\frac{r_{0}}{C_{L}}\right)-\mathrm{X}_{L} \quad X_{L}=\frac{\omega b}{2 C_{L}}\left(C_{L} / v-\cos \theta_{0}\right)
$$

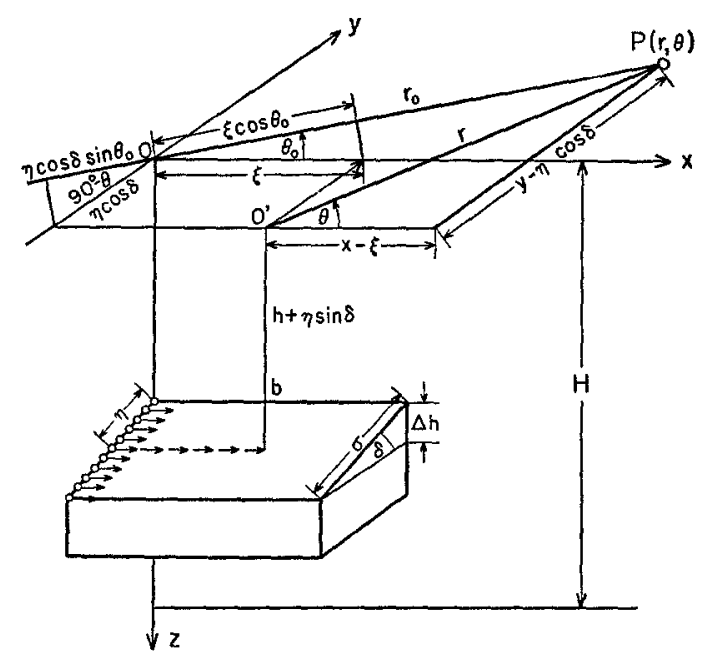

FIG. 23. Geometry of the strike-slip fault inside the layer.

$$
\begin{aligned}
\Delta_{1}{ }^{L} & =\frac{\omega \sigma}{2 C_{L}}\left\{\sin \theta_{0} \cos \delta+\alpha_{1} C_{L} \sin \delta\right\} \\
\Lambda_{2}{ }^{L} & =\frac{\omega \sigma}{2 C_{L}}\left\{\sin \theta_{0} \cos \delta-\alpha_{1} C_{L} \sin \delta\right\}
\end{aligned}
$$

For a vertical strike-slip fault we substitute $\delta=\pi / 2$ in (2-19) to get with

$$
\left.\begin{array}{c}
\Lambda_{1}{ }^{L}=\Lambda_{2}{ }^{L}=\frac{\omega \alpha_{1} \sigma}{2}=\Lambda^{L} \quad \sigma=h_{2}-h_{1} \\
U_{\theta}{ }^{L}=\frac{L}{\mu_{1} \sqrt{2 \pi}} \frac{\sin \theta_{0} N_{1}(\omega)}{\sqrt{r_{0} \cdot{ }_{r} \bar{K}}} \frac{\sin \mathrm{X}_{L}}{\mathrm{X}_{L}} \frac{\sin \Lambda^{L}}{\Lambda^{L}} \cos \left[\alpha_{1} \omega \frac{\left(h_{1}+h_{2}\right)}{2}\right] e^{i\left(\varphi_{L}+(3 \pi / 4)\right)}
\end{array}\right\}
$$

In order to generalize for a radiating dipole we differentiate (2-21) with respect to the coordinate $y$, using the operator $\partial / \partial y=\sin \theta_{0}\left(\partial / \partial r_{0}\right)+\left(\cos \theta_{0} / r_{0}\right)\left(\partial / \partial \theta_{0}\right)$ and neglecting terms higher than $r_{0}^{-1 / 2}$. We find that the relevant contribution to this order of magnitude comes from differentiating the exponent $e^{i \varphi_{L}}$. This means that in order to obtain the couple displacement from (2-21) we have to multiply it by $\omega / C_{L} \sin \theta_{0} e^{-(\pi i / 2)}$. Thus we have: 


$$
\left(U_{\theta}{ }^{L}\right)_{\text {couple }}=\frac{L}{\mu_{1}} \frac{\sin ^{2} \theta_{0}}{\sqrt{r_{0}}}{ }_{n} K^{1 / 2} N(\omega) \frac{\sin \mathrm{X}_{L}}{\mathrm{X}_{L}} e^{i\left(\varphi_{L}+(\pi / 4)\right)}+0\left[\left({ }_{n} K r_{0}\right)^{-3 / 2}\right]
$$

The layering-dependence of $U_{\theta}$ is given by:

$$
N_{1}(\omega)=\frac{\sin \Lambda^{L}}{\Lambda^{L}} \frac{N_{0}(\omega)}{\sqrt{2 \pi}} \cos \left[\alpha_{1} \omega \frac{\left(h_{1}+h_{2}\right)}{2}\right]
$$

Where the "dipole factor" is $\sin ^{2} \theta_{0}$, whereas we had the factor $\sin 2 \theta$ for the leading components of the Rayleigh dipole pattern The finiteness-factor $(\sin \mathrm{X} / \mathrm{X}) e^{i \varphi}$ is the same for both waves.

\subsection{TRANSFORMATION INTO THE TIME DOMAIN}

We shall now proceed to obtain a theoretical seismogram from (2-22). The same method will apply, mutatis mutandis, to any of the expressions in (2-13), (2-19), and (2-21).

First we note that the amplitude of $\left(U_{\theta}{ }^{L}\right)_{\text {couple }}$ in (2-22) is even in $\omega$. Taking the source time-dependence to be that of $\delta(t)$ we get:

$$
\left(U_{\theta}{ }^{L}\right)_{\text {couple }}(t)=\frac{L}{\pi \mu_{1}} \frac{\sin ^{2} \theta_{0}}{\sqrt{r_{0}}} \int_{0}^{\infty} G(\omega) \cos (\omega \tau+\pi / 4) d \omega
$$

with

$$
\begin{array}{rlrl}
\tau & =t-\frac{r_{0}}{C_{L}}-\frac{b}{2 C_{L}}\left(\frac{C_{L}}{v}-\cos \theta_{0}\right) \\
G(\omega) & ={ }_{n} K^{1 / 2} N_{1}(\omega) \frac{\sin \mathrm{X}_{L}}{\mathrm{X}_{L}}, & G(0)=0, G(\infty)=0
\end{array}
$$

and (2-24) can be integrated numerically for any observed dispersion curve $C_{L}=$ $C_{L}(\omega)$. This is only an approximation since our solution to the wave equation is exact only for a homogeneous mantle but is not an exact solution for the real earth. There is an apparent difficulty at the lower limit of the integral because we derived our previous theory on the assumption that $\omega r / C_{L} \gg 1$, thus restricting $\omega$ to non-vanishing values. However, the contribution to the integral from values of the integrand at the neighborhood of $\omega=0$ can be made small enough as compared with the rest of the integral.

Kelvin's stationary-phase method may be used to approximate (2-24). Using the results given by Pekeris (1948) and Newlands (1954) we have,

$$
\begin{gathered}
K_{0}=K\left(\omega_{0}\right), \quad C_{L}=C_{L}\left(\omega_{0}\right), \\
\text { for }\left(d^{2} K / d \omega_{0}{ }^{2}\right)>0, \text { and } \omega_{0}-\operatorname{root} \text { of }\left(d\left(\omega t-K r_{0}\right) / d \omega\right)=0: \\
U_{\theta}{ }^{L}(t) \approx \frac{2 L}{\mu_{1}} \frac{\sin ^{2} \theta_{0}}{r_{0}} \frac{G\left(\omega_{0}\right)}{\sqrt{2 \pi \mid \frac{d^{2} K}{d \omega_{0}^{2} \mid}}} \cos \left\{\omega_{0} t-r_{0} K_{0}-\frac{b \omega_{0}}{2 C_{L}}\left(\frac{C_{L}}{v}-\cos \theta_{0}\right)\right\}
\end{gathered}
$$

for $\left(d^{2} K / d \omega_{0}^{2}\right)<0$ : 


$$
U_{\theta}{ }^{L}(t)=\frac{2 L}{\mu_{1}} \frac{\sin ^{2} \theta_{0}}{r_{0}}-\frac{G\left(\omega_{0}\right)}{\sqrt{2 \pi \mid \frac{d^{2} K}{d \omega_{0}^{2} \mid}}} \sin \left\{\omega_{0} t-r_{0} K_{0}-\frac{b \omega_{0}}{2 C_{L}}\left(\frac{C_{L}}{v}-\cos \theta_{0}\right)\right\}
$$

and for $\left(d^{2} K / d \omega_{m}{ }^{2}\right)=0$, with $U_{m}$ the stationary value of group-velocity and $\omega_{m}$ the angular frequency corresponding to $U_{m}$ :

$$
\begin{aligned}
U_{\theta}{ }^{L}(t) \approx-\frac{4 L}{\mu_{1} \sqrt{2 \pi}} \frac{\sin ^{2} \theta_{0}}{r_{0}^{5 / 6}} \frac{G\left(\omega_{m}\right)}{\left[\frac{1}{2} \frac{d^{3} K}{d \omega_{m}^{3}}\right]^{1 / 3}} \\
\cdot A i(u) \cos \left\{\omega_{m} t-r_{0} K_{m}-\frac{b \omega_{m}}{2 C_{L}}\left(\frac{C_{L}}{v}-\cos \theta_{0}\right)+\frac{\pi}{4}\right\}
\end{aligned}
$$

where

$$
u=\frac{t-\frac{r_{0}}{U_{m}^{-}}}{\left[-\frac{1}{2} r_{0} \frac{d^{3} K}{d \omega_{m}^{3}}\right]^{1 / 3}}
$$

and where $A i(u)$ is the Airy-function defined by $A i(u)=\int_{0}^{\infty} \cos \left(u t+\frac{1}{3} t^{3}\right) d t$. The validity-condition for (2-28) was given by Pekeris (1948). The reader is reminded that our results apply only to the first $S H$ mode and for a single stationary point. In case of $n$ modes and $m$ roots of $d / d \omega\left(\omega t-{ }_{n} K r_{0}\right)=0$, a double sum is understood.

\subsection{APPLICATION TO THE G WAVE}

Our findings will be applied to the $G$ phase which is being repeatedly observed on records of major earthquakes. This phase appears on seismograms as a longperiod $S H$ pulse. It propagates with a rather constant group velocity of $4.38-4.44$ km/sec (Gutenberg and Richter 1934, 1936; Benioff 1958; Sato 1958; and Press 1959), and its power is concentrated mainly in the period range $40<T<400$. Gutenberg's (1959) low-velocity channel in the upper mantle provides a possible explanation to the low group-velocity of this phase.

Starting with an assumed constant group-velocity in the above-mentioned period range we integrate the basic relation $U_{0}=C-\lambda(d C / d \lambda)=U_{0}$ to get $C=\left(U_{0} / 1-\alpha_{0} T\right)$ (Sato 1958) with the observed values of $U_{0}=4.38-4.44 \mathrm{~km} / \mathrm{sec}$ and $\alpha_{0}=6 \times 10^{-4} \mathrm{sec}^{-1}$ (Sato 1958, Harkrider 1960). The wave number thus becomes a linear function in the angular frequency: $K(\omega)=1 / U_{0}\left(\omega-2 \pi \alpha_{0}\right)$. Being unable to use the stationary-phase approximation, we take the following alternative. We put the value of $K(\omega)$ directly into (2-24) and integrate between the limits of the spectral window from $\omega_{1}$ to $\omega_{2}$. It is observed that $G(\omega)$ in $(2-24)$ is a slowlyvarying function over the integration range. If we introduce into the integrand an exponential decay factor which governs the absorption in the real earth we have

$$
\begin{array}{r}
\left\{U_{\theta}{ }^{L}(t)\right\}_{n}=E_{0} \frac{\sin ^{2} \theta_{0}}{\sqrt{\Gamma_{0} \sin \Delta_{n}} G(\bar{\omega}) \int_{\omega_{1}}^{\omega_{2}} \omega^{p-1} e^{-\Omega \omega} \cos \left(\omega \tau_{0}+\delta_{0}\right) d \omega} \\
\bar{\omega}=\frac{\omega_{1}+\omega_{2}}{2} \quad \tau_{0}=t-\frac{b}{2 v}-\frac{1}{U_{0}}\left(r_{0}-\frac{b}{2} \cos \theta_{0}\right)
\end{array}
$$


$E_{0}$ is some real constant, $\Gamma_{0}$ is the radius of the Earth and the factor $\left(\Gamma_{0} \sin \Delta_{n}\right)^{-1 / 2}$ arises from the asymptotic expansion of the Legendre function of a large order, which replaces the Hankel function in problems of wave propagation over a sphere (Brune 1961, Brune, Nafe, and Alsop 1961, Yanovskaya 1958a). This asymptotic expansion is conditioned by $K \Gamma_{0} \gg 1 . \delta_{0}$ is a phase angle which includes the constant initial phase (Brune 1961) and the polar phase shift (Brune, Nafe and Alsop 1961). $\Omega=\Delta_{n} / 2 \bar{Q} U_{0}$, where $U_{0}$ is the constant value of the group velocity and $\bar{Q}$ is the average value of $Q$ (Sato 1958) over the spectral window. $n$ is the order of the surface wave and $\Delta_{n}$ is the distance along a great circle travelled by a surface wave of order $n . p$ is a characteristic of the source time-dependence. Since we are dealing with a horizontal couple we shall have $p=\frac{5}{2}$ for a delta source and $p=\frac{3}{2}$ for a step source. Equation (2-29) can be integrated numerically for every desired epicentral distance, and thus the corresponding wave form can be obtained. The exact evaluation of (2-29) is possible in terms of Incomplete Gamma-functions. Under the conditions $n>2, \tau_{0}>\left(T_{1} / 2 \pi\right)$, and $e^{-\Omega_{1}} \gg e^{-\Omega_{2}},(2-29)$ is approximated by

$$
\left\{U_{\theta}{ }^{L}(t)\right\}_{n} \approx \text { constant } \times \frac{\sin ^{2} \theta_{0}}{\sqrt{\Gamma_{0} \sin \Delta_{n}}} G(\bar{\omega}) \frac{\cos \left(\omega_{1} \tau_{0}+t^{-1} \frac{\tau_{0}}{\Omega}+\delta_{0}\right)}{\sqrt{\tau_{0}^{2}+\Omega^{2}}} e^{-\Omega \omega_{1}}
$$

The term $\operatorname{tg}^{-1}\left(\tau_{0} / \Omega\right)$ in $(2-30)$ is responsible for the broadening of the $G$ pulse due to absorption.

\section{Chapter 3-Derivation of the Source Parameters from the Spectrum of Surface Waves}

\subsection{INTRODUCTION}

Up to the present time, mantle Rayleigh waves and Love waves have been examined mainly by two methods. By the first method, dispersion curves are utilized to obtain information on the velocity distribution in the crust and the upper mantle of the earth (Ewing, Jardetzky, and Press 1957). The second method seeks information on the earthquake-source by the method of phase-equalization (Aki 1960). This method is based on the notion of a seismic point-source and thus a priori cannot provide us with any further knowledge which concerns itself with departures from this oversimplified model.

The theoretical results obtained in the former chapters can be used to obtain valuable data on real earthquake sources. This is done by introducing two new theoretical entities which are measurable on seismic records, whenever there exists a considerable departure from the point-source model. These are the directivity function and the differential-phase function.

\subsection{DERIVATION OF SOURCE PARAMETERS FROM THE AMPLITUDES}

It was shown in section 1.8 that in the case of a horizontally-moving fault, the amplitude ratio of two opposite-going components depends neither on the source spectrum nor on the layering, since these are cancelled out, while it does depend on the horizontal extension of the source, the velocity of rupture, and the strike of the 
fault with respect to the line joining the station with the initial epicenter. This function is henceforth called directivity, and is generally given by

$$
D=\left|\frac{\left(\frac{C}{v}+\cos \theta_{0}\right) \sin \frac{\pi b}{\lambda}\left(\frac{C}{v}-\cos \theta_{0}\right)}{\left(\frac{C}{v}-\cos \theta_{0}\right) \sin \frac{\pi b}{\lambda}\left(\frac{C}{v}+\cos \theta_{0}\right)}\right|
$$

where $C$ stands for either Rayleigh or Love phase-velocity, whichever is the case. In case the opposite-going components did not travel the same distance, they should be equalized in order to normalize the directivity which is defined for equal seismic paths. The directivity is defined for two different situations. In the first, the waves are studied at two different stations on opposite sides of the fault. The second situation is that in which surface waves of different order recorded at a single station. Such are for example mantle Rayleigh-waves or $G$ waves which start from the fault both in the direction of faulting and in the opposite direction and thus reach the recording station from two opposite directions. The directivity does not in practice depend on the direction of reception of the instrument (e.g., orientation of the strain seismograph). This happens because in the case of Love waves, $U_{r}{ }^{L}$ is of the order $1 /\left(K r_{0}\right)^{3 / 2}$, and for long ranges is negligible in comparison with $U_{\theta}{ }^{L}$. The same is true of the azimutal component $U_{\theta}{ }^{R}$ of the Rayleigh waves which for long ranges is negligible in comparison with the radial component $U_{r}{ }^{R}$. See equations (1-37) to (1-39) and (2-1) to (2-3). Thus the orientation of the instrument may affect the recorded amplitudes but will not affect the directivity. This argument does not hold for low values of $K r_{0}$, which is the case for very low frequencies.

The directivity is equal to unity for the case of a vertical dip-slip fault. It does not depend on the dip angle in case of a strike-slip fault with arbitrary dip angle and is not defined in the case of a dip-slip fault with arbitrary dip angle.

The source parameters $v$ and $b$ are derived from the seismic records in the following way. After digitizing the proper phases on the records and taking their numerical Fourier-transforms, the amplitude ratios for a set of selected frequencies are computed and then compared with the theoretical ratio given by (3-1), which has been corrected for absorption on different paths. The corrected amplituderatios are then plotted against the period and compared to theoretical trial-curves. These curves may easily be drawn by computing the theoretical zeros and infinities of (3-1). A wide enough spectrum (100-400 sec.) is needed to obtain good results for the three unknown parameters. A knowledge of the dependence of the phase velocity and the attenuation coefficient (or $Q$ ) on period are needed. $Q$ can be obtained independently using $G$ or mantle Rayleigh waves of the same parity.

\subsection{DERIVATION OF FAULT LENGTH FROM THE DIFFERENTIAL PHASE}

Consider a vertical strike-slip fault of length $b$ which extends vertically from depth $h_{1}$ to depth $h_{2}$ in an m-layered half-space. The total surface-wave radiation for the spectral frequency $\omega$ is given, according to $(2-22)$ and $\left(1-37^{a}\right),\left(1-38^{a}\right)$ and $\left(1-39^{a}\right)$ :

$$
U_{\theta}^{L}=\frac{L}{\mu_{1}} \frac{\sin ^{2} \theta_{0}}{\sqrt{r_{0}}} \sqrt{n} \bar{K} N_{m}(\omega) \frac{\sin \mathrm{X}_{L}}{\mathrm{X}_{L}} e^{i\left(\varphi_{L}+(\pi / 4)\right)}
$$




$$
\begin{aligned}
U_{r}^{R} & =\frac{L}{\mu_{1}} \frac{\sin 2 \theta_{0}}{\sqrt{r_{0}}} \sqrt{K_{n}} g_{r}{ }^{m}(\omega) \frac{\sin \mathrm{X}_{R}}{\mathrm{X}_{R}} e^{i\left(\varphi_{R}+(3 \pi / 4)\right)} \\
U_{z}^{R} & =\frac{L}{\mu_{1}} \frac{\sin 2 \theta_{0}}{\sqrt{r_{0}}} \sqrt{K_{n}} g_{z}^{m}(\omega) \frac{\sin \mathrm{X}_{R}}{\mathrm{X}_{R}} e^{i\left(\varphi_{R}+(3 \pi / 4)\right)}
\end{aligned}
$$

with

$$
\begin{aligned}
\mathrm{X}_{j} & =\frac{\omega b}{2 C_{j}}\left(\frac{C_{j}}{v}-\cos \theta_{0}\right) \\
\varphi_{j} & =\omega\left(t-\frac{r_{0}}{C_{j}}\right)-\mathrm{X}_{j} \quad j=L, R
\end{aligned}
$$

Equation (3-2) gives the Love-wave pattern while (3-3) and (3-4) represent the Rayleigh-wave pattern. The subscript $j$ in (3-5) and (3-6) refers to Love or Rayleigh waves, whichever is the case. The function $N_{m}$ has been derived for the simple case of a layer over a half-space in case of Love waves, and $g_{r}{ }^{m}$ and $g_{z}{ }^{m}$ have been given for a homogeneous half-space. The source is a horizontal dipole with harmonic time dependence. For an arbitrary time-dependence the layering functions $N_{m}$, $g_{r}{ }^{m}$ and $g_{z}{ }^{m}$ will be multiplied by a suitable factor. The situation for a single-layered half-space is displayed in figure 21 . We shall now apply these results to the propagation of mantle Rayleigh-waves and $G$ waves around the globe. Since at the moment we are concerned only with the phases of these waves, we need only add to (3-5) and (3-6) the polar shift $P_{n}$ (Brune, Nafe, and Alsop 1961) and replace $r_{0}$ by $\Delta_{n}$. Thus

$$
\begin{aligned}
\varphi_{n}=\omega\left(t_{0}-\frac{\Delta_{n}}{C}\right)-\frac{\omega b}{2 c}\left(\frac{C}{v}+(-)^{n} \cos \theta_{0}\right) & \pm 2 \pi m_{n}+P_{n} \\
n & =1,2, \cdots ; \quad 0 \leqq \theta_{0} \leqq 2 \pi ; \quad m=0,1,2, \cdots
\end{aligned}
$$

with

$$
\begin{aligned}
\Delta_{n} & =\Delta_{1}+\pi(n-1) \Gamma_{0}, & n & =1,3,5, \cdots \\
& =\pi n \Gamma_{0}-\Delta_{1}, & n & =2,4,6, \cdots \\
P_{n} & =\frac{\pi}{2}(n-1), & n & =1,2,3, \cdots
\end{aligned}
$$

$\Delta_{1}$ is the shortest distance from the source to the station. Equation (3-6) is the phase of a spectral component of a surface wave of order $n$ (time taken with respect to the origin time $t_{0}$ of the earthquake). $C$ is the phase velocity of either $G$ or $R$ waves, and $v$ is the velocity of rupture. The differential phase $\partial_{1} \varphi$ of two oppositegoing surface waves is therefore given by:

$$
\begin{aligned}
\partial_{1} \varphi=\frac{\varphi_{n+1}-\varphi_{n}}{2 \pi}=\frac{f}{c}\left(40,000-2 \Delta_{1}+b \cos \theta_{0}\right)+\left(M+\frac{1}{4}\right) & \\
M & =0,1,2, \cdots
\end{aligned}
$$

The slope of $\partial_{1} \varphi$ versus $f / C$ will yield the faulting length $b$ provided that $\theta_{0}$ and $C(f)$ are known. An alternative formula may be obtained by taking the differential 
phase of the second order $\partial_{2} \varphi$. Manipulating with three phases of three waves of consecutive orders, one finds:

$$
\partial_{2 \varphi}=\frac{\left(\varphi_{n+1}-\varphi_{n}\right)+\left(1-\frac{\Delta_{1}^{\circ}}{180^{\circ}}\right)\left(\varphi_{n+2}-\varphi_{n}\right)}{2 \pi}=f \frac{b}{C} \cos \theta_{0}+\text { constant }
$$

A special case of (3-9) and (3-10) is of interest for practical purposes of deriving the fault length without having recourse to the phase-velocity function. It is a well-established fact that the $G$ waves propagate on the flat portion of their groupvelocity curve, at least in the period range $150 \leqq T \leqq 250$ sec. Thus, solving the differential equation $U_{g}=$ const. $=U_{0}$ we obtain $f / C_{L}=f / U_{0}-\alpha_{0}$, where $\alpha_{0}$ is some constant. Therefore (3-10) reduces to

$$
\partial_{2} \varphi=f \frac{b}{U_{0}^{-}} \cos \theta_{0}+\text { constant }
$$

Furthermore, if each $G$ event is taken with respect to its own arrival time, we also have

$$
\partial_{1} \varphi=f \frac{b}{U_{0}} \cos \theta_{0}+\text { constant }
$$

which is more convenient to use when only two $G$ events are available, although less accurate, since the time of origin is known to a higher degree of accuracy than the arrival times of the individual events.

\section{Conclusions}

Our theory was able to explain the asymmetrical radiation pattern from earthquake faults and derive from it some important and previously elusive parameters of the source. It may serve to associate many earthquakes with at least two important parameters and thus be of great use in exploring the mechanism at the focus and the nature of the earth's interior. Moreover, the dependence of the surface-wave amplitudes on the vertical extent of the source were already indicated in most of the results of chapters 1 and 2 . In future work we intend to generalize our theory to fit the real earth and thus be able to derive the vertical extent of the faulting as well as its length. One should treat the derived quantities as rough values since their physical meaning is probably not sharply defined. We hope that future comparisons between measurements and the theory may point up ways to improve the theory, so as to cope with more realistic earthquake mechanisms.

Our present work has, however, another aspect which extends beyond its immediate applicability to the derivation of fault lengths and rupture velocities, and that is the notion that the classical point-source picture is no longer adequate as a model for most earthquakes. The finiteness and the motion of the source have their bearing on many measurements from earthquake records and ignoring them can lead to serious errors (e.g., calculations of phase- and group-velocities from readings of a single station, the concept of an "epicenter," etc.). Earthquake seismologists must from now on let this new dimension enter their way of thinking. 


\section{ACKNowledgMents}

Grateful acknowledgment is made to Professor Frank Press for his constant help and valuable advice in accomplishing this study. I have also had the benefit of stimulating discussions of this work with Professor H. Benioff, Dr. K. Aki and Dr. H. Honda. The author was Herbert Linden fellow during a portion of this research.

Partial support under contract No. AF-49 (638) 910 of the Air Force Technical Applications Center as part of the Advanced Research Projects Agency project Vela is also gratefully acknowledged.

\section{ReFarences}

Aki, K.

1960. "Study of Earthquake Mechanism by a Method of Phase Equalization," Jour, Geophys. Res., 65: 729-740.

Benioff, H.

1955. "Mechanism and Strain Characteristics of the White Wolf Fault as Indicated by the Aftershock Sequence," Calif. Div. Mines, Bull. 171, pp. 199-202.

1958. “Long Waves Observed in the Kamchatka Earthquake of November 4, 1952," Jour. Geophys. Res., 63: 589-593.

Brune, J. N.

1961. "Radiation Pattern of Rayleigh Waves from the Southeast Alaska Earthquake of July 10, 1958," Symposium on Fault Plane Studies (in press).

Brune, J. N., J. E. Nafe, and L. E. Alsop

1961. "The Polar Phase Shift of Surface Waves on a Sphere," Bull. Seism. Soc. Am., 51: $247-257$.

Ewing, M., W. Jardetzky, and F. Press

1957. Elastic Waves in Layered Media, McGraw-Hill Book Company, New York.

Gutenberg, B.

1955. "Magnitude Determination for Larger Kern County Shocks, 1952; Effects of Station Azimuth and Calculation Methods," Calif. Div. Mines, Bull. 171, pp. 171-175.

1959. "The Asthenosphere Low-Velocity Layer," Annali di Geofisica, 12: 439-460.

Gutenberg, B. and C. F. Richter

1934. "On Seismic Waves (First Paper)," Gerl. Beitr. zur Geophys., 43: 56-133.

1936. "On Seismic Waves (Third Paper)," Gerl. Beitr. zur Geophys., 47: 73-131.

Harkrider, D.

1960. Private Communication.

Knopoff, L. and F. A. Gilbert

1959. "Radiation from a Strike-Slip Fault," Bull. Seism. Soc. Am., 49: 163-178.

Lamb, H.

1916. "On Waves Due to a Travelling Disturbance," Phil. Mag., 13: 386-399 and 13: 539548 .

Morse, P. M. and H. Feshbach

1953. Methods of Theoretical Physics, Part II, McGraw-Hill Book Company, New York.

Newlands, $M$.

1954. “Lamb's Problem with Internal Dissipation, I," Jour. Acoustical Soc. Am., 26: 434448.

Pekeris, C. L.

1948. "Theory of Propagation of Explosive Sound in Shallow Water," in Geol. Soc. Am., Memoir or.

1955. "The Seismic Surface Pulse," Proc. Nat. Acad. Sci., 41: 469-470.

Press, F.

1959. "Some Implications on Mantle and Crustal Structure from $G$ Waves and Love Waves," Jour. Geophys. Res., 64: 565-568. 
Ryshik, I. M. and I. S. Gradstein

1957. Tables of Series, Products and Integrals, Veb. Deutscher Verlag der Wissenschaften, Berlin.

Sato, $\mathrm{Y}$.

1958. "Attenutation, Dispersion and the Wave Guide of the G Wave," Bull. Seism. Soc. Am., 48: 231-251.

Sezawa, K.

1929. "Generation of Rayleigh Waves from a Sheet of Internal Sources," Bull. Earthq. Inst., Tokyo University, 7: 417-435.

Sneddon, J.

1950. Fourier Transforms, McGraw-Hill Book Company, New York.

Yanovskaya, T. B.

1958. "On the Determination of the Dynamic Parameters of the Focus Hypocenter of an Earthquake from Records of Surface Waves, I," Izv. Akad. Nauk SSSR, Geophys. Ser., pp. 289-301.

1958a. "The Dispersion of Rayleigh Waves in a Spherical Layer," Izv. Akad. Nauk SSSR, Geophys. Ser., pp. 801-817.

Seismologicat Laboratory,

California Institute of Technology,

Pasadena, California

(Division of the Geological Seiences, contributions no. 995 and no. 1009.) 